\title{
An Optimal-Time Algorithm for Shortest Paths on Realistic Polyhedra
}

\author{
Yevgeny Schreiber
}

Received: 12 December 2007 / Revised: 3 January 2009 / Accepted: 7 January 2009 /

Published online: 30 January 2009

(C) Springer Science+Business Media, LLC 2009

\begin{abstract}
We generalize our optimal-time algorithm for computing (an implicit representation of) the shortest-path map from a fixed source $s$ on the surface of a convex polytope $P$ to three realistic scenarios where $P$ is a possibly nonconvex polyhedron. In the first scenario, $\partial P$ is a terrain whose maximum facet slope is bounded by any fixed constant. In the second scenario, $P$ is an uncrowded polyhedron-each axisparallel square $h$ of side length $l(h)$ whose smallest Euclidean distance to a vertex of $P$ is at least $l(h)$ is intersected by at most $O(1)$ facets of $\partial P$-an input model which, as we show, is a generalization of the well-known low-density model. In the third scenario, $P$ is self-conforming-here, for each edge $e$ of $P$, there is a connected region $R(e)$ of $O(1)$ facets whose union contains $e$, so that the shortest path distance from $e$ to any edge $e^{\prime}$ of $\partial R(e)$ is at least $c \cdot \max \left\{|e|,\left|e^{\prime}\right|\right\}$, where $c$ is some positive constant. In particular, it includes the case where each facet of $\partial P$ is fat and each vertex is incident to at most $O(1)$ facets of $\partial P$. In all the above cases the algorithm runs in $O(n \log n)$ time and space, where $n$ is the number of edges of $P$, and produces an implicit representation of the shortest-path map, so that the shortest path from $s$ to any query point $q$ can be determined in $O(\log n)$ time. The constants of proportionality depend on the various parameters (maximum facet slope, crowdedness, etc.). We also note that the self-conforming model allows for a major simplification of the algorithm.
\end{abstract}

Keywords Conforming subdivision · Continuous Dijkstra $\cdot$ Realistic polyhedral surface $\cdot$ Shortest path map $\cdot$ Terrain $\cdot$ Wavefront

Work on this paper was supported by NSF Grants CCR-00-98246 and CCF-05-14079, by a grant from the U.S.-Israeli Binational Science Foundation, by grant 155/05 from the Israel Science Fund, and by the Hermann Minkowski-MINERVA Center for Geometry at Tel Aviv University. The paper is based on the Ph.D. thesis of the author [18]. A preliminary version has been presented in Proc. of 23rd Annu. ACM Sympos. on Comput. Geom., pp. 74-83, 2007.

Y. Schreiber $(\bowtie)$

School of Computer Science, Tel Aviv University, Tel Aviv 69978, Israel

e-mail: yevgeny.schreiber@cs.tau.ac.il 


\section{Introduction}

\subsection{Background}

The algorithmic problem of determining the Euclidean shortest path on the surface of a polyhedron between two points, or, more generally, computing a compact representation of all such paths that emanate from a fixed source point, has been studied for more than 20 years. In this paper we extend the recent algorithm of Schreiber and Sharir [19], which solves the problem (optimally) for a convex polytope, into more realistic scenarios.

The problem on a convex polytope $P$ with $n$ vertices in $\mathbb{R}^{3}$ was first solved by Sharir and Schorr [22], who gave an $O\left(n^{3} \log n\right)$-time algorithm; soon afterwards, Mount [15] improved the running time to $O\left(n^{2} \log n\right)$. For a general, possibly nonconvex polyhedral surface, O'Rourke et al. [17] gave an $O\left(n^{5}\right)$-time algorithm; subsequently, Mitchell et al. [13] presented an $O\left(n^{2} \log n\right)$ algorithm, extending the technique of Mount [15]. All algorithms in [13, 15, 22] use the same general approach, called "continuous Dijkstra," first formalized in [13]. The technique keeps track of all the points on the surface whose shortest path distance to the source $s$ has the same value $t$, and maintains this "wavefront" as $t$ increases. Finally, Chen, and Han [5] solved the problem, for a general polyhedron, in $O\left(n^{2}\right)$ time, using a rather different approach - they build a shortest path sequence tree, using an observation that they call "one angle one split" to bound the number of branches.

The problem has been more or less "stuck" after Chen and Han's paper, and the quadratic time barrier seemed very difficult to break. ${ }^{1}$ Recently, Schreiber and Sharir [19] have presented an optimal $O(n \log n)$-time algorithm for the case where $P$ is convex. The algorithm uses the continuous Dijkstra approach and follows the general outline of the technique of Hershberger and Suri [9], which solves, in $O(n \log n)$ time, a closely related problem: Compute the shortest path map from a fixed source point on the plane, in the presence of polygonal obstacles whose total number of vertices is $n$; this map can be used to answer single-source shortest path queries in $O(\log n)$ time.

However, the question whether the more general case of a nonconvex polyhedron has a subquadratic solution, has remained open. The known approaches to this problem either use approximate solutions (see $[2,3,8,11,12,26]$ ) or focus on special cases in which various "realistic" assumptions on the input are being made [14]. The latter approach has also been used to solve other geometric problems [1, 4, 6, 7, 20, 23-25]; some of these input models are discussed and compared to each other by de Berg et al. [7].

In this paper, we present three realistic models, for each of which the problem can be solved, in $O(n \log n)$ time, by an appropriate extension of the algorithm of [19]. These models were listed in the abstract and are discussed in more detail in Sect. 3. The common feature of these models is that they all admit variants of a

\footnotetext{
${ }^{1}$ In 1999 Kapoor [10] has announced an algorithm that computes a shortest path from the source $s$ to a single target point $t$ on an arbitrary polyhedral surface in $O\left(n \log ^{2} n\right)$ time. However, as far as we know, the details of Kapoor's algorithm have not yet been published.
} 
so-called conforming surface subdivision of $\partial P$, a major tool used in the algorithm of [19] (following the idea of the so-called free space subdivision of [9]). Informally (see [9, 19] and below for details), this subdivision consists of artificial "transparent" edges, which are sufficiently separated from one another, and are the "atoms" on which the continuous Dijkstra propagation is applied. Interestingly, once such a subdivision is shown to exist and to be efficiently constructible, the rest of the algorithm and its analysis are very similar (albeit not identical) to those in [19].

We will briefly review below the algorithm of [19] (and of [9]), but, since both algorithms (and their analysis) are fairly complex, we need to rely on the results of these papers and assume some familiarity of the reader with them.

\subsection{Preliminaries}

The input to the problem is a three-dimensional polyhedron $P$ with a connected boundary, having $n$ vertices, and a source point $s \in \partial P$. Without loss of generality, we assume that $s$ is a vertex of $P$, that all facets of $P$ are triangles, and that no edge of $P$ is axis-parallel. (All these properties can be enforced in $O(n)$ time, or, if some of the original facets are nonsimple, in $O(n \log n)$ time.)

Most of the definitions below are imported from [19] (where some of them were borrowed in turn from [13, 21, 22]).

Let $\phi, \phi^{\prime}$ be facets of $\partial P$ that share a common edge $\chi$. The unfolding of $\phi^{\prime}$ onto (the plane containing) $\phi$ is the rigid transformation that maps $\phi^{\prime}$ into the plane containing $\phi$, effected by an appropriate rotation about the line through $\chi$, so that $\phi$ and the image of $\phi^{\prime}$ lie on opposite sides of that line. Let $\mathcal{F}=\left(\phi_{0}, \phi_{1}, \ldots, \phi_{k}\right)$ be a sequence of distinct facets such that $\phi_{i-1}$ and $\phi_{i}$ have a common edge $\chi_{i}$ for $i=1, \ldots, k$. We say that $\mathcal{E}=\left(\chi_{1}, \chi_{2}, \ldots, \chi_{k}\right)$ is the corresponding edge sequence of $\mathcal{F}$. The unfolding transformation $U_{\mathcal{E}}$ is the transformation of 3-space that represents the rigid motion that maps $\phi_{0}$ to the plane of $\phi_{k}$, through a sequence of unfoldings at the edges $\chi_{1}, \chi_{2}, \ldots, \chi_{k}{ }^{2}$ The plane of $\phi_{k}$ is called the destination plane of $U_{\mathcal{E}}$.

A geodesic path $\pi$ is a simple (that is, non-self-intersecting) path along $\partial P$ that is locally optimal, in the sense that, for any two sufficiently close points $p, q \in \pi$, the portion of $\pi$ between $p$ and $q$ is the unique shortest path that connects them on $\partial P$. Such a path $\pi$ is always piecewise linear, and it bends only at edges or vertices of $P$; its length is denoted as $|\pi|$. For any two points $a, b \in \partial P$, a shortest geodesic path between them is denoted by $\pi(a, b)$ (for certain degenerate locations of $a, b$, there may be more than one such path).

We call a vertex $v$ of $P$, such that the sum of the angles at $v$ on the facets adjacent to $v$ is greater than $2 \pi$, a stepping-stone vertex, or, in short, an s-vertex. It is shown in [13] that a geodesic path goes through an alternating sequence of s-vertices and (possibly empty) edge sequences such that the unfolded image of the path along any such edge sequence (between two consecutive s-vertices) is a straight line segment. The general form of a shortest geodesic path is similar, with the additional restrictions

\footnotetext{
${ }^{2}$ That is, for $i=1, \ldots, k$, let $\varphi_{i}$ be the transformation that unfolds $\phi_{i-1}$ to the plane of $\phi_{i}$ about $\chi_{i}$. The unfolding $U_{\mathcal{E}}$ is then the composed transformation $\varphi_{k} \circ \cdots \circ \varphi_{1}$. However, in what follows, we will also use $U_{\mathcal{E}}$ to denote the collection of all partial unfoldings $\varphi_{k} \circ \cdots \circ \varphi_{i}$ for $i=1, \ldots, k$.
} 
Fig. 1 Regions of $\operatorname{SPM}(s)$ are bounded by the bisectors (the solid straight segments and hyperbola branches) and by the shortest paths from $s$ to vertices of $P$ (dashed)

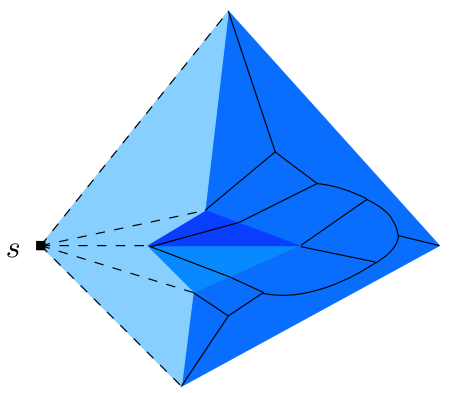

that no edge can appear in more than one edge sequence and each edge sequence must be simple. Assuming general position, two shortest geodesic paths from $s$ can overlap each other at a common prefix that ends at an s-vertex, or meet at a common final destination, but otherwise they are disjoint. For convenience, the word "geodesic" is understood as a default for all paths under consideration and is omitted in the rest of the paper.

The shortest path distance between $x$ and $y$ for $x, y \in \partial P$ is denoted as $d(x, y)$ (we use this notation as well when $x, y$ are points on a plane and $d(x, y)=|x y|$ ); we also put $\delta(x)=d(s, x)$. We denote by $d_{3 D}(x, y)$ the Euclidean distance in $\mathbb{R}^{3}$ between $x$ and $y$.

A point $z \in \partial P$ is called a ridge point if there exist at least two distinct shortest paths from the source $s$ to $z$. The shortest path map with respect to $s$ (see Fig. 1), denoted $\operatorname{SPM}(s)$, is a subdivision of $\partial P$ into $O(n)$ connected regions whose interiors are vertex-free and contain neither ridge points nor points belonging to shortest paths from $s$ to vertices of $P$; for each such region $R$, there is only one shortest path $\pi(s, p)$ to any $p \in R$, which also satisfies $\pi(s, p) \subset R$. For a pair of such adjacent regions $R_{1}, R_{2}$, denote by $s_{1}$ (resp., $s_{2}$ ) the last s-vertex in the shortest path from $s$ to any internal point of $R_{1}$ (resp., $R_{2}$ ); it is possible that $s_{1}, s_{2}$, or both are equal to $s$. A boundary between $R_{1}, R_{2}$ is either a shortest path to a vertex of $P$ or a bisector $b\left(s_{1}, s_{2}\right)$, which is the locus of points equidistant from $s$ via $s_{1}$ and $s_{2}$. The unfolded image of this locus is a branch of a hyperbola (which can degenerate into a straight line ${ }^{3}$ ), so that for each point $p \in b\left(s_{1}, s_{2}\right)$, we have $\delta\left(s_{1}\right)+d\left(s_{1}, p\right)=\delta\left(s_{2}\right)+d\left(s_{2}, p\right)$.

Overview and Organization As in [19], our algorithm for computing (an implicit representation of) $\operatorname{SPM}(s)$ consists of two phases. The first phase, which is discussed in Sects. 2 and 3, is the construction of a conforming surface subdivision of $P$ (defined in the following section). The construction of [19] is briefly reviewed, in a somewhat more general form, in Sect. 2 to emphasize the properties that make it possible to extend it for nonconvex input models. Section 3 introduces two such realistic models, in which these properties hold, and an even more restrictive (but still natural) input model, which allows for a major simplification of the algorithm. In Sect. 4 we review the second phase of the shortest path algorithm, namely, the

\footnotetext{
${ }^{3}$ In general position, straight bisectors arise only when the last s-vertices on the two alternative paths (either of which may be $s$ itself) are equal.
} 
Dijkstra-style wavefront propagation, and describe the adjustments of the algorithm that are needed for the above input models. We close in Sect. 5 with a discussion, which includes the extension to the construction of so-called geodesic Voronoi diagrams (as in [19]), and with several open problems.

Perhaps the most striking feature of the extension of the algorithm is that it depends mainly on the existence of a conforming surface subdivision of $P$. Once such a subdivision is shown to exist (and to be efficiently computable), the actual wavefront propagation is a routine adaptation of [19] (although it does require several nontrivial adjustments). A considerable portion of this paper is therefore devoted to the analysis and construction of suitable conforming surface subdivisions.

\section{The Conforming Surface Subdivision Revisited}

We briefly review, in a somewhat more general form, the construction of the conforming surface subdivision for convex polytopes, and its predecessor, the conforming subdivision for planar polygonal regions, of Hershberger and Suri [9], since we use, as in [19], a three-dimensional analog of this ingredient.

An $\alpha$-conforming subdivision $S_{2 D}$ is an axis-parallel quad-tree-style subdivision of the plane, into $O(n)$ cells, constructed on the vertices of the obstacles. (In [9], $S_{2 D}$ is used to construct a more complex subdivision of the free space that involves also the obstacle edges; we briefly describe it later in Sect. 3, where it becomes relevant.) Each cell of $S_{2 D}$ is a square or a square-annulus bounded by $O(\alpha)$ straight line edges (called transparent edges), which are subsegments of the edges of the square or of its hole, and contains at most one obstacle vertex; each transparent edge $e$ satisfies the following crucial well-covering property:

$\left(\mathbf{W C}_{2 D}\right)$ There is a region $R(e)$, called the well-covering region of $e$, which consists of only $O(1)$ cells and contains $e$ in its interior, so that, for any transparent edge $e^{\prime}$ on $\partial R(e)$ or outside $R(e), d\left(e, e^{\prime}\right) \geq \alpha \max \left\{|e|,\left|e^{\prime}\right|\right\}$.

The $\alpha$-conforming subdivision $S_{2 D}$ is constructed in [9] in two phases: First, a 1-conforming subdivision $S_{2 D}^{1}$ is constructed; then, each edge of $S_{2 D}^{1}$ is subdivided into $\alpha$ sub-edges (in [9], $\alpha=2$ ). The subdivision $S_{2 D}^{1}$ is constructed in a bottom-up fashion by simulating a growth process of an axis-parallel square box around each obstacle vertex until their union becomes connected; see [9] for details.

In [19], we similarly construct an axis-parallel three-dimen sional $\alpha$-conforming subdivision $S_{3 D}$ (we use $\alpha=16$ ): First, a three-dimensional 1-conforming subdivision $S_{3 D}^{1}$ is constructed by simulating a growth process of a cube box around each vertex of $P$, and then each face of $S_{3 D}^{1}$ is subdivided into $\alpha \times \alpha$ square subfaces. (We use the term "facet" when referring to a triangle of $\partial P$ and the term "face" when referring to the square faces of the three-dimensional cells of $S_{3 D}^{1}$. Furthermore, we say that each such face is subdivided into square "subfaces" in $S_{3 D}$.) Each 3D-cell of the resulting subdivision $S_{3 D}$ is either a whole cube or a perforated cube (with a single cube-shaped hole); it is bounded by $O\left(\alpha^{2}\right)$ square subfaces and contains at most one vertex of $P$. Each subface $h$ satisfies the following version of the well-covering property (we say that $h$ is well covered): 
Fig. 2 The well-covering region of the darkly shaded face $h$ contains, in this example, a total of 39 cells (nine transparent large cells on the back, five lightly shaded large cells on the front, and 25 small cells, also on the front)

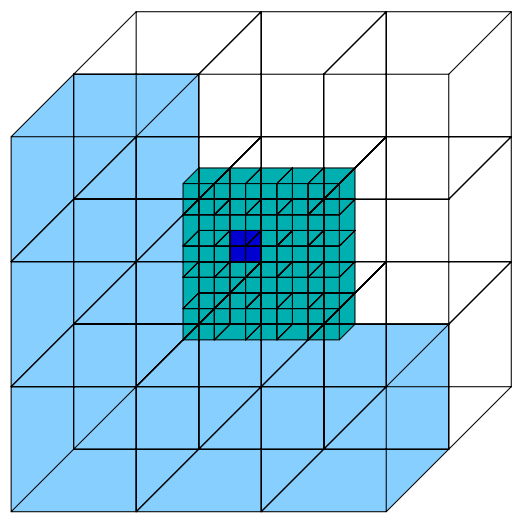

(WC $\mathbf{W}_{3 D}$ ) For any subface $h^{\prime} \in S_{3 D}$ such that $h$ and $h^{\prime}$ are portions of nonadjacent faces of $S_{3 D}^{1}, d_{3 D}\left(h, h^{\prime}\right) \geq \alpha \max \left\{l(h), l\left(h^{\prime}\right)\right\}$, where $l(h)$ is the side length of $h$. (See Fig. 2.)

It follows from $\left(\mathrm{WC}_{3 D}\right)$ that there is a well-covering region $R(h)$ of only $O(1)$ 3D-cells that contains $h$ in its interior, so that, for each subface $h^{\prime}$ on $\partial R(h)$ or outside $R(h), d_{3 D}\left(h, h^{\prime}\right) \geq \alpha \max \left\{l(h), l\left(h^{\prime}\right)\right\}$.

$S_{3 D}$ also satisfies the following minimum vertex clearance property: ${ }^{4}$

(MVC) For any vertex $v$ of $P$ and for any subface $h$ of $S_{3 D}, d_{3 D}(h, v) \geq \frac{\alpha}{4} l(h)$.

As described in [19], the construction of $S_{3 D}$ differs from the construction of $S_{2 D}$, except for modifying some of the steps for the extension from two to three dimensions, only by the following simple modification needed to satisfy (MVC): Around each vertex of $P$, we construct a cube of side length $\frac{1}{16}$ that is not part of $S_{3 D}$ (we assume that the smallest $L_{\infty}$-distance between any pair of vertices of $P$ is 1 ), and all the following steps of the construction ensure that these cubes are kept far enough from the outer boundaries of the larger cells that are "grown" around these cubes; see [19] for details.

When $S_{3 D}$ is constructed, we implicitly compute, in $O(\log n)$ time, the edge sequences of $\partial P$ that are intersected by each subface of $S_{3 D}$, using a surface unfolding data structure, which is described later in this section.

Intersection Connectivity and Intersection Ratio We continue the description of the construction in [19], using several structural parameters, whose values for the case of a convex polytope are obvious, but they will assume different values when we extend the construction to other kinds of polyhedra. Denote by $\kappa$ the maximum number of connected components of the intersection $\partial P \cap h$ taken over all subfaces $h$

\footnotetext{
${ }^{4}$ This is not to be confused with the closely related minimum clearance property of the subdivision $S_{2 D}$ in [9]. 
Fig. 3 A subface $h$ and three maximal connected portions $\xi_{1}, \xi_{2}, \xi_{3}$ that constitute the intersection $h \cap \partial P$
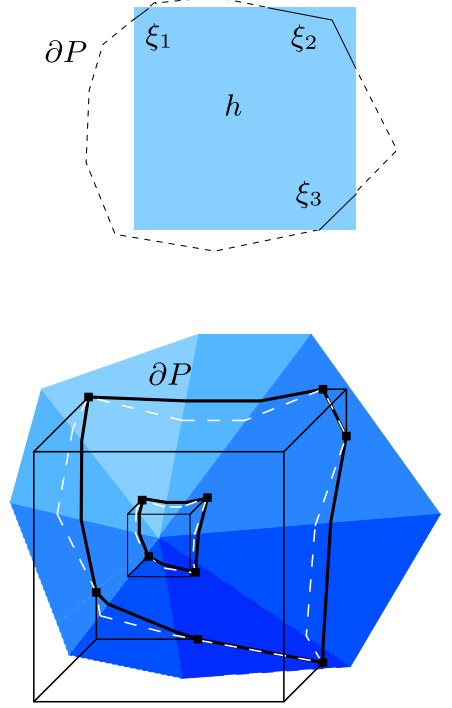

Fig. 4 The intersection components between $\partial P$ (shaded) and the subfaces of $S_{3 D}$ (bounded by thin solid lines) are dashed. The transparent edges are the thick solid (geodesic) paths between the endpoints of the corresponding intersection components. (In this example the transparent edges do not cross each other)

of $S_{3 D}$; we call $\kappa$ the intersection connectivity of $P$. Denote by $\rho$ the maximum intersection ratio $|\xi| / l(h)$ taken over all subfaces $h$ of $S_{3 D}$ and over all components $\xi$ of $\partial P \cap h$ (where $|\xi|$ denotes the length of $\xi$ ). For a convex $P, \kappa \leq 4$ and $\rho \leq 4$ (see Fig. 3).

For each subface $h$ of $S_{3 D}$ and for each connected component $\xi$ of the intersection $\partial P \cap h$, we implicitly compute the shortest geodesic path $e$ between the endpoints of $\xi$ that traverses the same edge sequence of $\partial P$ as $\xi$; see Fig. 4 (again, we emphasize that the current description refers only to the case where $P$ is convex). We call $e$ a transparent edge, and, after splitting the transparent edges at the points where they intersect each other (we show in Lemma 2.2 below that there are at most $O\left(\kappa \alpha^{2}\right)$ such intersections for each $e$ ), we call the resulting partition of $\partial P$ by transparent edges a conforming surface subdivision $S$. The subdivision $S$ consists of $O(n)$ surface cells, each of which contains at most one vertex of $P$ and is bounded by $O(1)$ transparent edges (a more detailed analysis follows momentarily). Each transparent edge $e$ satisfies the following variant of the well-covering property (we say that $e$ is well covered) - note that this variant, as opposed to $\left(\mathrm{WC}_{3 D}\right)$, does not depend on $\alpha$ :

$\left(\mathbf{W C}_{S}\right)$ There is a well-covering region $R(e)$ of only $O(1)$ cells of $S$ that contains $e$ in its interior, so that, for any transparent edge $e^{\prime}$ on $\partial R(e), d\left(e, e^{\prime}\right) \geq$ $2 \max \left\{|e|,\left|e^{\prime}\right|\right\}$.

The following three lemmas prove the properties of $S$ that are listed above. Each of these lemmas has a corresponding (but less general) equivalent in [19]; nevertheless, we present the proofs here to demonstrate the dependence on $\kappa$ and $\rho$ (and $\alpha$ ).

Lemma 2.1 If $\rho \leq \alpha / 4$, then no vertex of $P$ can be incident to a transparent edge; that is, for each transparent edge, its unfolded image is a straight segment. 
Proof By (MVC), for any vertex $v$ of $P$ and for any subface $h$ of $S_{3 D}, d_{3 D}(h, v) \geq$ $\frac{\alpha}{4} l(h)$. Let $e$ be a transparent edge originating from the connected component $\xi$ of the intersection $\partial P \cap h$ for some subface $h$ of $S_{3 D}$. Then $|e| \leq|\xi|$ by the definition of transparent edges, and $|\xi| \leq \rho l(h) \leq \frac{\alpha}{4} l(h)$. Since each endpoint $u$ of $e$ is contained in $h$, it satisfies

$$
d_{3 D}(u, v) \geq d_{3 D}(h, v) \geq \frac{\alpha}{4} l(h) \geq|\xi| \geq|e| ;
$$

hence $e$ cannot reach any vertex $v$ of $P$.

Lemma 2.2 If $\rho<\alpha$, then each transparent edge that originates from a subface (in $S_{3 D}$ ) of a face $F \in S_{3 D}^{1}$ meets at most $O\left(\kappa \alpha^{2}\right)$ other transparent edges that originate from subfaces of faces adjacent to $F$ (or from $F$ itself), and does not cross any other transparent edges (that originate from subfaces of faces not adjacent to $F$ ).

Proof Let $e, e^{\prime}$ be transparent edges originating from the respective cuts $\xi=\partial P \cap h$, $\xi^{\prime}=\partial P \cap h^{\prime}$, where $h, h^{\prime}$ are subfaces of $S_{3 D}$, and let $F \in S_{3 D}^{1}$ be the face that contains $h$. Since both endpoints of $e$ are in $h$, for any point $p \in e$, we have $d_{3 D}(p, h) \leq$ $\frac{1}{2}|e| \leq \frac{1}{2}|\xi| \leq \frac{1}{2} \rho l(h)$. Similarly, for any point $q \in e^{\prime}, d_{3 D}\left(q, h^{\prime}\right) \leq \frac{1}{2} \rho l\left(h^{\prime}\right)$. If $h, h^{\prime}$ are subfaces of faces that are not adjacent in $S_{3 D}^{1}$, then, since $h$ and $h^{\prime}$ are well covered, it follows that $d_{3 D}\left(h, h^{\prime}\right) \geq \alpha \max \left\{l(h), l\left(h^{\prime}\right)\right\}$; hence the three-dimensional distance between any point of $e$ and any point of $e^{\prime}$ is at least $(\alpha-\rho) \max \left\{l(h), l\left(h^{\prime}\right)\right\}$, and therefore, if $\rho<\alpha, e$ does not intersect $e^{\prime}$. There are only $O(1)$ faces in $S_{3 D}^{1}$ that are adjacent to $F$, and each of them is subdivided into $O\left(\alpha^{2}\right)$ subfaces in $S_{3 D}$. Each of these subfaces induces $O(\kappa)$ transparent edges (as follows from the definition of $\kappa$ ), and the claim of the lemma follows. ${ }^{5}$

Lemma 2.3 If $\kappa$ is a constant and $\rho \leq \alpha / 3$, then each transparent edge of $S$ is well covered.

Proof Consider an original transparent edge $e^{\star}$ (before the splitting of intersecting transparent edges) that originates from the intersection component $\xi$ of $\partial P \cap h$ for some subface $h$ of $S_{3 D}$. The endpoints of $e^{\star}$ are incident to $h$, which is well covered in $S_{3 D}$ by a region $R(h)$ consisting of $O(1)$ 3D-cells. We define the well-covering region $R(e)$ of every edge $e$, derived from $e^{\star}$ by splitting, as the connected component containing $e$, of the union of the surface cells that originate from the 3D-cells of $R(h)$. Since each 3D-cell of $S_{3 D}$ induces at most $O\left(\kappa \alpha^{2}\right)$ transparent edges, each of which intersects at most $O\left(\kappa \alpha^{2}\right)$ other transparent edges, and since each such pair of crossing transparent edges form at most one new surface cell, there are $O\left(\kappa^{2} \alpha^{4}\right)=$ $O(1)$ surface cells in $R(e)$. Since all the surface cells that are incident to $e$ originate

\footnotetext{
${ }^{5}$ Moreover, it is easy to check that the claim is stronger for a convex $P$, where all subfaces in a single face induce a total of $O(\kappa)$ transparent edges. Other observations that improve the upper bound on the total number of transparent edges can be made for some of the realistic models discussed in this paper, but, since they do not affect the asymptotic complexity of our algorithm, we omit them for the sake of simplicity.
} 
from 3D-cells that are incident to $h$ and therefore are in $R(h), R(e)$ is not empty, and its interior contains $e$.

For each transparent edge $e^{\prime}$ originating from a subface $g$ that lies on the boundary of $R(h), d(h, g) \geq d_{3 D}(h, g) \geq \alpha \max \{l(h), l(g)\}$. The length of $e$ satisfies $|e| \leq\left|e^{\star}\right| \leq|\xi| \leq \rho l(h)$, and, similarly, $\left|e^{\prime}\right| \leq \rho l(g)$. Therefore, for each point $p \in e$, we have $d_{3 D}(p, h) \leq \frac{1}{2}|e| \leq \frac{1}{2} \rho l(h)$, and for each point $q \in e^{\prime}$, we have $d_{3 D}(q, g) \leq \frac{1}{2} \rho l(g)$. Hence, for each $p \in e, q \in e^{\prime}$, we have

$$
d(p, q) \geq d_{3 D}(p, q) \geq(\alpha-\rho) \max \{l(h), l(g)\} \geq \frac{\alpha-\rho}{\rho} \max \left\{|e|,\left|e^{\prime}\right|\right\} .
$$

Therefore, if $\rho \leq \frac{\alpha}{3}$, we have $\frac{\alpha-\rho}{\rho} \geq 2$ and $d\left(e, e^{\prime}\right) \geq 2 \max \left\{|e|,\left|e^{\prime}\right|\right\}$.

So far, our analysis holds for any $P$; the following constructions are less general.

The Surface Unfolding Data Structure We review here this structure for the case where $P$ is a convex polytope, as in [19]. Later, we discuss its extension for nonconvex polyhedra. In order to construct $S$ and then to process it into a Riemann structure described below, we need the following surface unfolding data structure for $P$, which can process each of the following types of queries in $O(\log n)$ time:

(i) Given an axis-parallel subface $h$ of $S_{3 D}$ (or, more generally, any axis-parallel rectangle), compute all the connected components of the (convex) curve $\partial P \cap h$ and represent these components in compact form (without computing $\partial P \cap h$ explicitly).

(ii) Given $h$ as above, perform a binary search over the segments in any connected component of $\partial P \cap h$ (using the linear order of the segments along $\partial P \cap h$ ).

(iii) Given $h$ as above and given a pair of edges $\chi, \chi^{\prime}$ of $P$ such that the points $\chi \cap h, \chi^{\prime} \cap h$ lie in a common connected component $\xi \subseteq \partial P \cap h$, compute the unfolding transformation $U_{\mathcal{E}}$, where $\mathcal{E}$ is the polytope edge sequence intersected by $\xi$ between $\chi$ and $\chi^{\prime}$ (inclusive).

The surface unfolding data structure is constructed, in $O(n \log n)$ time, as follows. We sort the vertices of $P$ in ascending $z$-order and sweep a horizontal plane $\zeta$ upwards through $P$. At each height $z$ of $\zeta$, the cross section $P(z)=\zeta \cap \partial P$ is a (convex) polygon, which we represent as a (persistent) search tree $T_{z}$ constructed on its vertices. (We break $P(z)$ at, say, its leftmost vertex, thereby linearizing the sequence of its vertices.) We construct, in a completely symmetric fashion, two additional trees $T_{x}$ and $T_{y}$ by sweeping $P$ with planes orthogonal to the $x$-axis and to the $y$-axis, respectively. This allows us to process a query of type (i) or (ii) in $O(\log n)$ time, see [19] for details. To process a query of type (iii), we precompute and store the unfolding $U_{v}$ of the sequence $\mathcal{E}_{v}$ of polytope edges stored at the leaves of the subtree of $v$ for each node $v$ of $T_{x}, T_{y}, T_{z}$. This can be easily done in a bottom-up fashion, as described in detail in [19]. We can then answer a query of type (iii) by composing the prestored transportations along search paths of the trees, see [19] for full details.

For a general nonconvex polyhedron $P$, we still want to be able to perform these kinds of queries. However, this construction does not carry over to the general case, 
because it assumes that the segments of $\partial P \cap h$ are linearly ordered (and that the order is easy to determine, e.g., by slope) for any axis-parallel rectangle $h$; we describe the necessary changes, which produce a variant of this structure for certain classes of nonconvex polyhedra $P$, in Sect. 3 .

The Riemann Structures This structure is also reviewed here for the case of a convex polytope $P$; its extensions to more general polyhedra are discussed later in the paper.

As explained in more detail in Sect. 4, the continuous Dijkstra paradigm simulates a unit-speed wavefront expanding from the source point $s$ and spreading along the surface of the polytope; at simulation time $t$, the true wavefront consists of points whose shortest path distance to $s$ along $\partial P$ is $t$. Once $S$ is constructed, the transparent edges form the elements on which the Dijkstra-style propagation is performed: At each step, the wavefront is ascertained to have completely covered some transparent edge $e$ (that is, each point in $e$ is crossed by a shortest path of length $t$ encoded in $W$ ) and is then advanced toward (a constant number of) nearby transparent edges that have not yet been ascertained to be completely covered. However, to implement the propagation of a wavefront through a cell $c$ of $S$, we have to handle effectively (i) the possibility that shortest paths may traverse $c$ in "homotopically inequivalent" ways (e.g., by going around a vertex of $P$ in $c$ or a hole of $c$ in two different ways) and (ii) the possibility of overlap between distinct portions of $c$ when unfolded onto some common plane. To overcome these difficulties, we introduce in [19] a Riemann structure that efficiently represents the unfolded regions of $\partial P$. This representation subdivides each surface cell into $O(1)$ simple building blocks of the following four types (see Fig. 5); a planar unfolding of such a block (i) is unique, and (ii) is a simply connected polygon (that does not overlap itself) bounded by $O(1)$ straight line segments (each of which is an unfolded image of (a portion of) either a transparent edge or an edge of $\partial P)$.

Type I For each facet $\phi$ of $\partial P$, any connected component $B$ of $c \cap \phi$ that contains an endpoint of a transparent edge of $\partial c$ in its closure is a building block of type I of $c$.

Type II Let $v$ be the (only) vertex of $P$ in $c$, let $e$ be a transparent edge in $\partial c$, and let $\mathcal{F}$ be a maximal sequence of adjacent facets that are incident to $v$ and $e$, but neither to endpoints of $e$ nor to any other transparent edge between $e$ and $v$. Then the union $B$, over all facets $\phi \in \mathcal{F}$, of the portion of $\phi$ between $e \cap \phi$ and $v$ is a building block of type II of $c$.

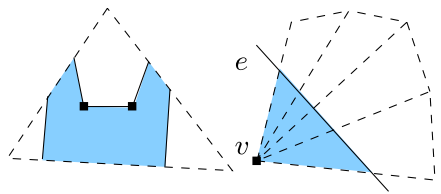

(a)

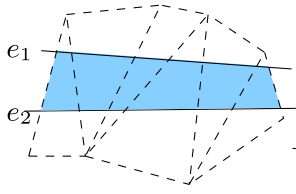

(c)

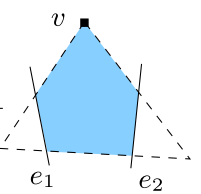

(d)

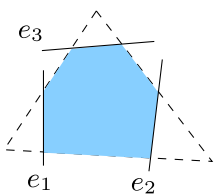

(e)

Fig. 5 Building blocks (shaded) of types: (a) I, (b) II (unfolded), (c) III (unfolded), and (d), (e) IV 
Fig. 6 (a) A surface cell $c$ containing a single vertex of $P$ and bounded by four transparent edges (solid lines) is partitioned here into ten building blocks (whose shadings alternate).

(b) The tree $T_{B}(e)$ of building blocks of $c$, where $B$ is a block bounded by the (thick) transparent edge $e$

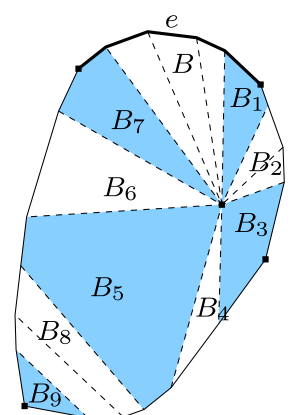

(a)

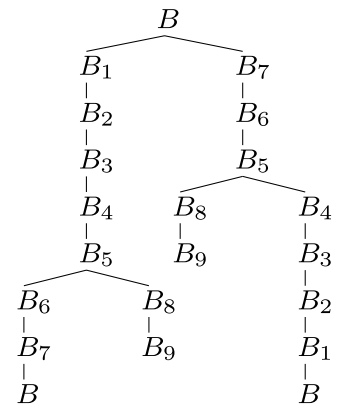

(b)

Type III Let $e_{1}, e_{2}$ be two distinct transparent edges in $\partial c$, and let $\mathcal{F}$ be a maximal sequence of adjacent facets that are incident to $e_{1}$ and $e_{2}$, but neither to their endpoints nor to any other transparent edge between $e_{1}$ and $e_{2}$. Then the union $B$, over all facets $\phi \in \mathcal{F}$, of the portion of $\phi$ between $e_{1} \cap \phi$ and $e_{2} \cap \phi$ is a building block of type III of $c$.

Type IV Let $\phi$ be a facet of $\partial P$. Any connected component $B$ of the region $c \cap \phi$ that does not contain an endpoint of any transparent edge and whose boundary contains a portion of each of the three edges of $\phi$ is a building block of type IV of $c$.

Let $e$ be a transparent edge on the boundary of some surface cell $c$, and let $B$ be a building block of $c$ so that $e$ appears on its boundary. The block tree $T_{B}(e)$ is a rooted tree whose nodes are building blocks of $c$, which is defined recursively as follows. The root of $T_{B}(e)$ is $B$. Let $B^{\prime}$ be a node in $T_{B}(e)$. Then its children are the blocks $B^{\prime \prime}$ that satisfy the following conditions:

1. $B^{\prime}$ and $B^{\prime \prime}$ are adjacent through a common portion of a polytope edge on their boundaries.

2. $B^{\prime \prime}$ does not appear as a node on the path in $T_{B}(e)$ from the root to $B^{\prime}$, except possibly as the root itself (that is, we allow $B^{\prime \prime}=B$ if the remaining conditions are satisfied).

3. If $B^{\prime \prime}=B$, then (a) it is of type II or III (that is, if a root is a building block of type I or IV, it cannot appear as another node of the tree), and (b) it is a leaf of the tree.

We denote by $\mathcal{T}(e)$ the set of all block trees $T_{B}(e)$ of $e$ (constructed from the building blocks of both cells containing $e$ on their boundaries), and we call it the Riemann structure of $e$. Indeed, the unfolding of all the blocks in a tree (with their multiple appearances) may overlap itself, but our propagation algorithm will ignore these overlaps, treating them as different layers of a Riemann surface. We show in [19] that it takes $O(n \log n)$ total time to construct the Riemann surfaces $\mathcal{T}(e)$ for all transparent edges $e$, using the surface unfolding data structure.

We also define, for each vertex $v$ which is either $s$ itself or an s-vertex, a similar Riemann structure $\mathcal{T}(v)$ consisting of all the block trees $T_{B}(v)$ whose root is a building block $B$ incident to $v$. (In [19] this variant is described for $v=s$.) 
We say that $c$ is punctured if either $c$ is not simply connected, so its boundary consists of more than one cycle, or $c$ contains a vertex $v$ of $P$ in its interior (here $v$ is regarded as an artificial singleton hole of $c$, even if $v$ is an s-vertex, which can, potentially, be traversed by shortest paths). We call $v$ and/or the inner cycles of $\partial c$ the islands of $c$. Denote by $G$ the set that includes the unique vertex $v \in c$ (if it exists) and all the transparent edges in $\partial c$, and let $X, Y$ be two distinct elements of $G$. For a pair of points $x_{1}, x_{2} \in X$ and a pair of points $y_{1}, y_{2} \in Y$, let $\pi\left(x_{1}, y_{1}\right), \pi\left(x_{2}, y_{2}\right)$ be a pair of geodesic paths that connect $x_{1}$ to $y_{1}$ and $x_{2}$ to $y_{2}$, respectively, within $c$. If $v$ exists and is an s-vertex, we make the additional assumption that neither $\pi\left(x_{1}, y_{1}\right)$ nor $\pi\left(x_{2}, y_{2}\right)$ contains $v$ in its relative interior. We say that $\pi\left(x_{1}, y_{1}\right)$ and $\pi\left(x_{2}, y_{2}\right)$ are homotopic in $c$ with respect to $X$ and $Y$ if one path can be continuously deformed into the other within $c$, while their corresponding endpoints remain in $X$ and $Y$, respectively. (In particular, none of the deformed paths can pass through $v$, although a path can start or end at $v$.) When $c$ is punctured, the geodesic paths that connect, within $c$, points in $X$ to points in $Y$ can fall into several different homotopy classes, depending on the way in which these paths navigate around the islands of $c$.

Let $\mathcal{B}$ be a path (of building blocks) in $T_{B}(e) \in \mathcal{T}(e)$ that connects $e$ to some transparent edge $f \in \partial c$ or to the vertex $v \in c$. Then all shortest paths that reach $f$ or $v$ from $e$ via the building blocks in $\mathcal{B}$ belong to the same homotopy class. Moreover, we show in [19] that for each such homotopy class $H$ of shortest paths from one transparent edge of $\partial c$ to another, or from a transparent edge to a vertex of $P$, there is a sequence of building blocks $\mathcal{B}$ in some block tree of $\mathcal{T}(e)$ so that the union of the blocks of $\mathcal{B}$ contains every path in $H$. In the nonconvex case, a similar property holds, provided that the paths are not allowed to go through any s-vertex. Moreover, this property also holds for block trees $T_{B}(v) \in \mathcal{T}(v)$ for the s-vertices $v$.

Note that, in the case of a general nonconvex $P$, efficient construction of the Riemann structures is problematic, since it relies on availability of the surface unfolding data structure. Moreover, as already discussed, we face additional difficulties when the cell $c$ contains an s-vertex $v$. In this case, a shortest path that traverses $c$ and passes through $v$ is not properly encoded in the block trees of $c$, because these trees only encode paths that do not go through vertices. Such a path should be regarded as the concatenation of two subpaths, ending and starting at $v$, respectively; each of these subpaths is "captured" separately, within the appropriate Riemann structure. We describe the changes needed to handle this situation for a nonconvex $P$ in Sect. 3.

Further details of the shortest path algorithm that are relevant to the extension to the cases where $P$ is nonconvex are described in Sect. 4.

\section{Models of Realistic Polyhedra}

We start this section by introducing two models of nonconvex polyhedra for which the maximum intersection ratio $\rho$ and the intersection connectivity $\kappa$ are constants. This property allows us to set $\alpha$ to a constant value that satisfies Lemmas 2.1-2.3, which, combined with an efficient construction of the surface unfolding data structure (described below for each of the models), yields an efficient construction of the 
surface subdivision for each of these input models. Since $\rho$ and $\kappa$ will be constants in the models that we discuss, the dependence of the performance of the algorithm on $\rho$ and $\kappa$ (or, rather, on $\alpha$, which can also be chosen to be a constant) is not analyzed, nor is it stated explicitly. As will be shown in the rest of the paper, the time and space complexity of the algorithm, for any of the models considered in this paper, is comparable with that of [19], that is, it is $O(n \log n)$.

\subsection{Terrains with Bounded Facet Slopes}

The most intuitive case discussed in this paper is that in which $P$ is a terrain, that is, the graph of a continuous piecewise linear bivariate function (so any line parallel to the $z$-axis meets $P$ in at most one point). For a facet $\phi$ of $P$, the slope of $\phi$ is the acute dihedral angle between the plane containing $\phi$ and the $x y$-plane (equivalently, it is the acute angle between the normal of $\phi$ and the $z$-axis). It is easy to see that if the maximum facet slope (over all facets of $P$ ) is bounded by some constant $\beta<\pi / 2$, then, for any straight segment $\chi$ that is fully contained in a facet of $P$, we have

$$
1 \leq \frac{|\chi|}{|\tilde{\chi}|} \leq \gamma:=\frac{1}{\cos \beta},
$$

where $\tilde{\chi}$ is the projection of $\chi$ onto the $x y$-plane (see Fig. 7). ${ }^{6}$

For the terrain model, we construct a version of the 3-dimensional conforming subdivision $S_{3 D}$ that does not contain horizontal faces, so that each 3D-cell of $S_{3 D}$ is an unbounded vertical prism whose $x y$-cross section is either a square or a squareannulus. To do so, we construct the projection $\tilde{S}$ of $S_{3 D}$ onto the $x y$-plane, as described next, and then extend each of its cells to the corresponding vertical prism.

Denote by $\tilde{P}$ the projection of $P$ onto the $x y$-plane. If the boundary of $\tilde{P}$ is an axis-parallel rectangle or if $\tilde{P}$ is the whole $x y$-plane, then, clearly, the intersection

Fig. 7 The slope of the facet $\phi$ is at most $\beta$

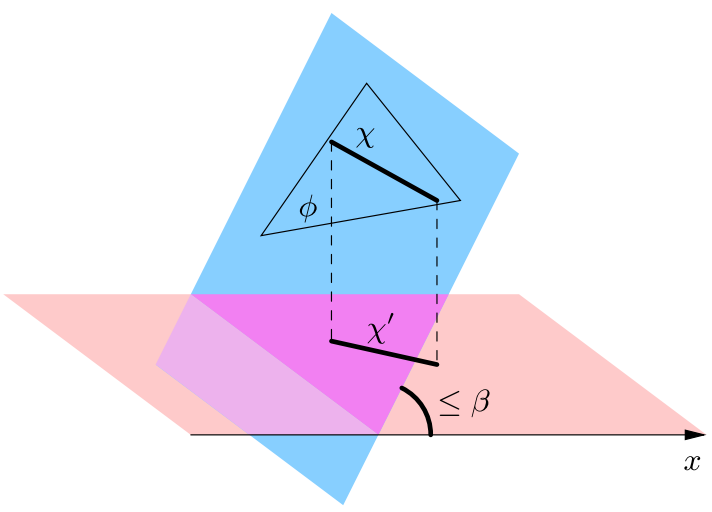

\footnotetext{
${ }^{6}$ Note that, for convenience, our definition differs from the conventional definition of slope, which is usually the tangent of that angle. Nevertheless, our slope is bounded by some constant $\beta<\pi / 2$ if and only if the conventional slope is bounded (by some other constant).
} 
Fig. 8 The intersection connectivity of $P$ with a vertical strip $h$ is $\kappa=1$
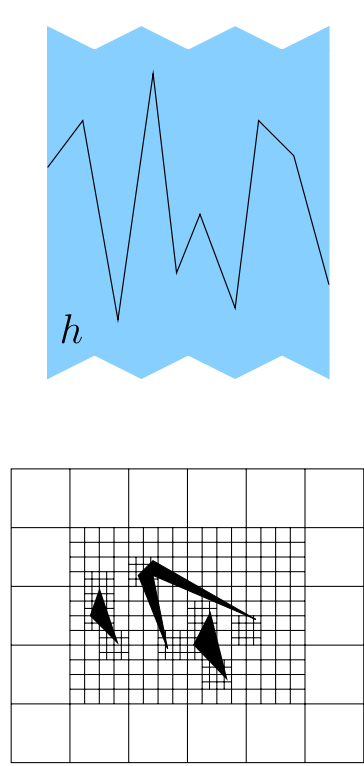

Fig. 9 The "obstacles" and the planar conforming subdivision of the free space ignoring the nonboundary edges of $\tilde{P}$

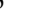


by lifting each edge of $\tilde{S}_{2 D}$ to the vertical subface of $S_{3 D}$ that it spans; by the construction of [9], no transparent edge of $\tilde{S}_{2 D}$ intersects an obstacle edge, and therefore the intersection connectivity of $S_{3 D}$ is $\kappa=1$. Denote by $l(h)$ the length of the crosssection of such a subface $h$ of $S_{3 D}$ with the $x y$-plane. For each intersection $\xi=P \cap h$, we have $|\xi| \leq \gamma l(h)$, since $\xi$ consists of straight segments, each of which is at most $\gamma$ times longer than its $x y$-projection. Thus $\rho \leq \gamma$. It is easy to see that the new definitions of $S_{3 D}$ and of $l(h)$ do not violate any property needed for Lemmas 2.1-2.3, so the lemmas continue to hold.

Remark The assumption that the maximum facet slope is bounded is not too restrictive-even if $\beta$ is reasonably large, $\gamma$ will still be small enough; for example, for $\beta=0.45 \pi$, we have $\gamma<6$.4. In fact, if $\beta \leq 0.419 \pi \approx 75.52^{\circ}$, then $\gamma<4$ and $\alpha=\lceil 4 \gamma\rceil \leq 16$, so the complexity of $S_{3 D}$ is similar to that in the case where $P$ is a convex polytope.

The Surface Unfolding Data Structure Similarly to [19], we construct the surface unfolding data structure of $P$ which can process each of the following types of query in $O(\log n)$ time:

(i) Given an (axis-parallel vertical strip) subface $h$ of $S_{3 D}$ (or, more generally, any axis-parallel vertical strip), compute the (connected) polygonal line $\partial P \cap h$ and represent it in compact form (without computing it explicitly).

(ii) Given $h$ as above, perform a binary search over the segments in $\partial P \cap h$ (using the order of the segments along $\partial P \cap h)$.

(iii) Given $h$ as above, and given a pair of edges $\chi, \chi^{\prime}$ of $P$ intersected by $h$, compute the unfolding transformation $U_{\mathcal{E}}$, where $\mathcal{E}$ is the polytope edge sequence intersected by $h$ between $\chi$ and $\chi^{\prime}$ (inclusive).

The surface unfolding data structure consists of the trees $T_{x}, T_{y}$, which are defined and constructed similarly to those in [19] (note that we do not need the tree $T_{z}$ in the current scenario). Using this surface unfolding data structure and $S_{3 D}$, we can construct the conforming surface subdivision $S$ in $O(n \log n)$ time, as (briefly) described in Sect. 2; the rest of the details are identical to those in [19].

Riemann Structures As shown in [19], we can use the surface unfolding data structure to construct the Riemann structures $\mathcal{T}(e)$ for all transparent edges $e$ in a total of $O(n \log n)$ time. The construction here is essentially identical to that in [19]. However, as noted in Sect. 2, these structures do not encode all shortest paths that traverse the respective cells of $S$. That is, if a cell $c$ contains an s-vertex $v$, then paths that go through $v$ require special treatment. Expanding upon this note, observe that the portion $\pi \cap c$ of a shortest path $\pi$ that enters $c$ through some transparent edge $e$ and passes through $v$, is a concatenation of a path $\pi_{1}$ that reaches $v$ and a path $\pi_{2}$ from $v$ to a point where it leaves $c$ (or to its endpoint if it lies in $c$ ). The path $\pi_{1}$ enters $c$ through $e$ and does not pass through a vertex in $c$, so its portion $\pi_{1} \cap c$ belongs to a homotopy class whose corresponding block sequence is a path in a tree of $\mathcal{T}(e)$; therefore, we only need to consider all paths that leave $v$.

To do so, for each cell $c$ that contains an s-vertex $v$, we construct, in addition to the Riemann structures $\mathcal{T}(e)$ for all transparent edges $e \in \partial c$, the Riemann structure 
$\mathcal{T}(v)$ that consists of the block trees $T_{B}(v)$ (defined similarly to trees $T_{B}(e)$, that is, satisfying conditions (1)-(3) in Sect. 2) for all building blocks $B$ that contain $v$ on their boundaries. As mentioned earlier, this construction is not new-we have used it in [19] for the Riemann structure $\mathcal{T}(s)$. As shown there, $\mathcal{T}(s)$ can be constructed in $O(\log n)$ time, and the block sequences in its block trees represent all the homotopy classes of shortest paths from $s$ to the transparent edges on the boundary of its surface cell. Hence, applying the same procedure to each s-vertex of $P$, the total time needed to construct all the Riemann structures $\mathcal{T}(v)$ and $\mathcal{T}(e)$ for all s-vertices $v$ and transparent edges $e$ is $O(n \log n)$.

This completes the description of the construction of a conforming surface subdivision $S$ and the Riemann structures for a terrain with bounded facet slope. We will describe in Sect. 4 how these structures are used by the propagation algorithm, to achieve overall running time $O(\log n)$.

\subsection{Uncrowded Polyhedra}

Let $k$ be a positive integer, and let $\mathcal{S}$ be a set of polyhedral objects in $\mathbb{R}^{3}$. For an axis-parallel square $h$, denote by $L(h)$ the minimum Euclidean distance from $h$ to a vertex of an object of $\mathcal{S}$. We call the set $\mathcal{S} k$-crowded if any axis-parallel square $h$ of side length $l(h)$ for which $l(h) \leq L(h)$ is intersected by at most $k$ objects of $\mathcal{S}$. If $k$ is a small constant, we say that $\mathcal{S}$ is uncrowded.

We assume that $\partial P$ is a $k$-crowded scene of triangles; see Fig. 10. For each axisparallel square $h$ for which $l(h) \leq L(h), \partial P \cap h$ consists of at most $k$ straight segments, each of which is at most $\sqrt{2}$ times longer than $l(h)$; therefore $\rho \leq \sqrt{2} k$, and the intersection connectivity $\kappa$ is at most $k$. Hence we can construct the three-dimensional conforming subdivision $S_{3 D}$, as described in Sect. 2, using $\alpha=\lceil 4 \sqrt{2} k\rceil$.

The Surface Unfolding Data Structure and the Riemann Structures Since in the current scenario the segments of $\partial P \cap h$ for a subface $h \in S_{3 D}$ are not necessarily ordered, and even if they are, it is not easy to manipulate this order (e.g., to compare two segments), we cannot construct the surface unfolding data structure in the same way as in the case of a convex $P$. Instead, for each subface $h$, the property (MVC) guarantees that $L(h)$ (that is, the smallest distance from $h$ to a vertex of $P$ ) is at least $\frac{\alpha}{4} l(h) \geq \sqrt{2} k \cdot l(h)$. Hence $h$ satisfies the requirement in the definition of $k$-crowdedness, so at most $k$ triangles intersect $h$. We can therefore directly find (details to be given shortly) at most $k$ facets that intersect $h$ (and store them in a list), so the overall storage is $O(k n)$. With these lists precomputed, we can process any query of type (i)-(iii) (as described in Sect. 2) in $O(k)$ time. (Note that we could

Fig. 10 For a $k$-crowded polyhedron $P$, each axis-parallel square $h$ whose closest vertex of $\partial P$ is at distance at least $l(h)$ from $h$ is intersected by at most $k$ facets of $\partial P$

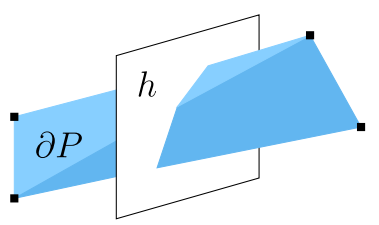


have simply regarded each intersection of a facet with $h$ as a separate transparent edge, simplifying the algorithm; however, this approach produces (up to $k$ times) more transparent edges and therefore adds steps to the continuous Dijkstra algorithm.)

Moreover, we can find all such intersections in $O(k n)$ time as follows. For each facet $\phi$ of $\partial P$, we choose one of its vertices, $v$; denote by $c_{3 D}$ the (whole cube) $3 \mathrm{D}$-cell that contains $v$. In $O(1)$ time we compute the intersection of $\phi$ with the boundary of $c_{3 D}$, and, in particular, we find the $O(1) 3 \mathrm{D}$-cells that are adjacent to $c_{3 D}$ and intersect $\phi$. For each of these $O(1) 3 \mathrm{D}$-cells, we continue in the same manner, computing the intersection of its boundary with $\phi$ in $O(1)$ time and continuing into adjacent 3D-cells. Since we spend only $O(1)$ time for each intersection of $\phi$ with a subface and since there is a total of at most $k n$ such intersections for all facets $\phi$, it takes only $O(k n)$ time to compute them all.

Note that, while doing this, we can also compute the value of $\rho$ exactly by summing the lengths of the intersections of each face of $S_{3 D}^{1}$ with facets of $\partial P$-this lets us use the potentially smaller value $\alpha=\lceil 4 \rho\rceil$ when we partition each face of $S_{3 D}^{1}$ into $\alpha \times \alpha$ subfaces. (Observe that the assumptions of Lemmas 2.1-2.3 are still satisfied, since $\rho \leq \alpha / 4$.)

Using this data structure, we construct the conforming surface subdivision $S$ as described in Sect. 2 and the Riemann structures $\mathcal{T}(v)$ and $\mathcal{T}(e)$ for all s-vertices $v$ and transparent edges $e$, as in Sect. 3.1.

\subsection{Relation to Other Models}

We have considered two families of polyhedra, terrains of bounded facet slope and uncrowded polyhedra, which share a common property: The intersection of each subface $h$ of a three-dimensional conforming subdivision $S_{3 D}$ with the polyhedron surface consists of at most $O(1)$ connected components, each of which is only $O(1)$ times longer than $l(h)$. It is unclear whether this property is really necessary for an efficient solution of the shortest path problem on a polyhedral surface; however, it shows that for a large class of polyhedra, the problem is not more difficult than for a convex surface (and therefore, as will follow from the description in Sect. 4, can be solved in optimal time by our algorithm).

Uncrowded polyhedra resemble the uncluttered model of de Berg [6], where each axis-parallel cube that does not contain a vertex of an axis-parallel bounding box of any object in the scene, intersects at most $O(1)$ objects. In spite of the similarity, however, one can easily find an example of an uncrowded polyhedron that is not uncluttered (e.g., if there is an axis-parallel cube $c$ as in Fig. 11(a) that contains a vertex of an object, but not a vertex of an axis-parallel bounding box of any object in the scene, so that $c$ is intersected by many objects), as well as uncluttered polyhedra that are not uncrowded (e.g., consider an axis-parallel cube $c$ as in Fig. 11(b) that contains a vertex of an axis-parallel bounding box of an object, but not a vertex of any object in the scene, so that $c$ is intersected by many objects).

The clutter factor of de Berg's model is also interesting because of its relation to another well-known geometric parameter, the density [6, 7, 20, 23, 24]. We call 


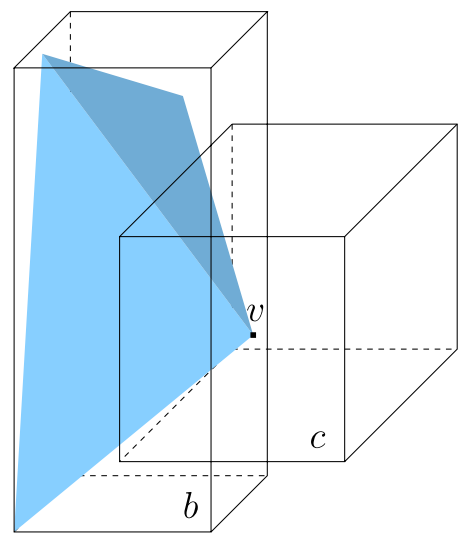

(a)

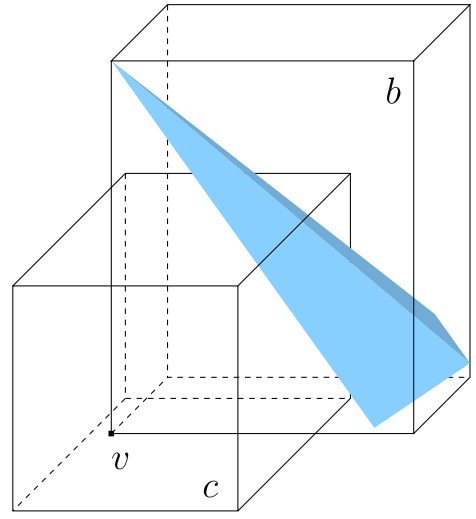

(b)

Fig. 11 An example of an axis-parallel cube $c$ that contains (a) a vertex $v$ of an object but not a vertex of its axis-parallel bounding box $b$, or (b) a vertex $v$ of the bounding box $b$ of an object but none of the object vertices

an object $R$-large if the radius of its minimum enclosing ball is at least $R$; then the density of a scene is the maximum number of $R$-large objects that intersect a ball of radius $R$. If the density of the scene is a small constant, it is called a low-density scene. De Berg shows in [6] that a low-density scene must be uncluttered. In the following lemma, we show that if the density of the facets of a polyhedron $P$ is low, $P$ must be uncrowded.

Lemma 3.1 If the density of the facets of a polyhedron $P$ is $\lambda$, then $P$ is a $\lambda$-crowded polyhedron.

Proof Let $h$ be an axis-parallel square whose distance to each vertex of $P$ is at least $l(h)$; in particular, for each facet $\phi$ of $\partial P$ that intersects $h$, the distance from each vertex of $\phi$ to $h$ is at least $l(h)$. Therefore, for each such $\phi$, the distance from any point $p \in \phi \cap h$ to each of the vertices of $\phi$ is at least $l(h)$. This implies that the radius of the minimum enclosing circle of $\phi$ (in the plane that contains $\phi$ ) is at least $l(h)$. Indeed, let $C$ denote the minimum enclosing circle of $\phi$, and suppose to the contrary that its radius is smaller than $l(h)$. Let $C^{\prime}$ be a copy of $C$ (within the same plane) centered at some point $p \in \phi \cap h$. Then $C^{\prime}$ does not contain any vertex of $\phi$, so all three vertices lie in the "crescent" $C \backslash C^{\prime}$ (see Fig. 12). It is then elementary to show that $p$ lies outside $\phi$, contradicting the construction.

Denote by $B$ the ball of radius $l(h)$ centered at the center of $h$. Clearly, $h$ is contained in $B$, and therefore any facet of $\partial P$ that intersects $h$ must intersect $B$. Since the density of the facets is $\lambda$, no more than $\lambda$ facets whose minimum enclosing ball radius is at least $l(h)$ intersect $B$, and therefore $h$ is intersected by at most $\lambda$ facets of $\partial P$.

The converse of Lemma 3.1 is not true, as shown in the following lemma. 
Fig. 12 The "crescent" $C \backslash C^{\prime}$ (shaded) contains all the three vertices of the triangle $\phi$, a contradiction to the fact that $p$ (which is the center of $C^{\prime}$ ) lies in $\phi$

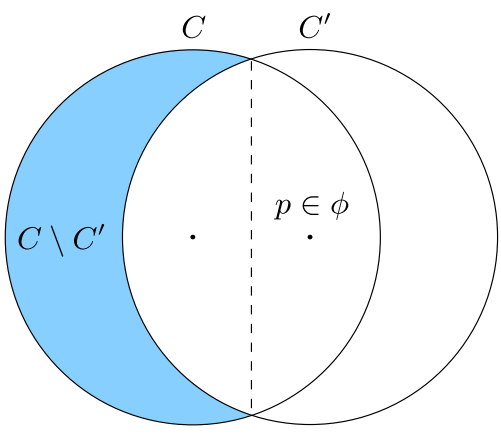

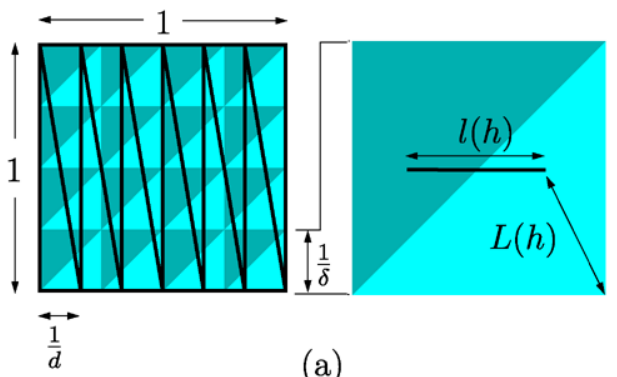

(a)

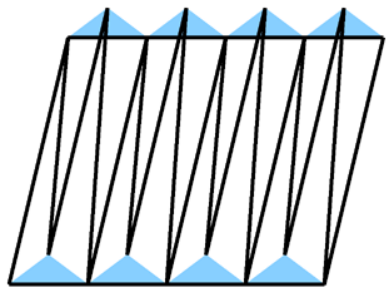

(b)

Fig. 13 (a) The projections $\tilde{\mathcal{L}}$ (alternatingly shaded) and $\tilde{\mathcal{U}}$ (solid segments). (b) The facets of $\mathcal{S}$ (shaded) connect $\mathcal{U}$ and $\mathcal{L}$

Lemma 3.2 For any integers $\lambda \geq 5$ and $\mu \geq 1$, there is a $\lambda$-crowded polyhedron $P$ whose facet density is greater than $\mu$.

Proof Given $\lambda$ and $\mu$, let $P$ be an "almost flat" polyhedron whose boundary contains an upper hull $\mathcal{U}$, a lower hull $\mathcal{L}$, and a set $\mathcal{S}$ of facets parallel to the $x z$-plane that connect $\mathcal{U}$ and $\mathcal{L}$, as described below. All vertices of $\mathcal{U}$ (resp., $\mathcal{L}$ ) lie on or very close above (resp., below) the $x y$-plane. The projection $\tilde{\mathcal{U}}$ of $\mathcal{U}$ onto the $x y$-plane is a sequence $\mathcal{R}$ of $d=\lceil(\mu+1) / 2\rceil$ congruent axis-parallel rectangles, each of which is composed of two triangles. The boundary of $\tilde{\mathcal{U}}$ is the unit square $Q$ (centered at the origin), so each rectangle in $\mathcal{R}$ is of size $1 \times 1 / d$. The projection $\tilde{\mathcal{L}}$ of $\mathcal{L}$ onto the $x y$-plane is $Q$, subdivided into $\delta \times \delta$ sub-squares, each of which is composed of two triangles, so that

$$
\delta>\left\lceil\frac{2 d(\sqrt{7}-1)}{3(\lambda-4)}\right\rceil .
$$

See Fig. 13(a) for an illustration. The boundary of $\tilde{\mathcal{L}}$ lies on $\mathcal{L}$ (in three dimensions, on the plane $z=0$ ), and all the $y$-parallel edges of $\tilde{\mathcal{U}}$ are contained in $\mathcal{U}$. The set $\mathcal{S}$ is composed of two subsets, each of which contains $d$ facets that connect an $x$-parallel edge of $\tilde{\mathcal{U}}$ with the facets of $\mathcal{U}$ above that edge. See Fig. 13(b).

The density of the facets of $\mathcal{U}$ (and therefore, of $P$ ) is at least $2 d>\mu$ (consider a ball of radius $1 / 2$ centered at the origin-it intersects all the triangles of $\mathcal{U}$ ). To 
compute the crowdedness of the facets of $P$, consider an axis-parallel square $h$. It is easy to see that, in order to intersect as many as possible facets of $P$ while keeping $l(h) \leq L(h), h$ must intersect the long edges of $\mathcal{U}$. However, since the edges of $\mathcal{U}$ lie very close to the vertices of $\mathcal{L}$, it is easy to verify that the maximal $l(h)$ that is not greater than $L(h)$ is $(\sqrt{7}-1) /(3 \delta)$ (so that the projection of $h$ onto the $x y$-plane is a segment "in the middle" of a sub-square of $\tilde{\mathcal{L}})$. Such $h$ intersects at most $2 d \cdot l(h)+2$ facets of $\mathcal{U}$ and at most two facets of $\mathcal{L}$, so the total number of facets of $P$ intersected by $h$ is $2 d(\sqrt{7}-1) /(3 \delta)+4<\lambda$.

A model that is closely related to (but is far less general than) the terrain with bounded facet slope is introduced by Moet et al. [14]. There, except for requiring the maximal facet slope to be bounded, three additional assumptions are made: (1) The projection $\tilde{P}$ of $P$ onto the $x y$-plane has low density; (2) the boundary of $\tilde{P}$ is a rectangle; and (3) the maximal ratio of lengths of any pair of edges of $\tilde{P}$ is a constant. It is shown in [14] that, under all four assumptions, the explicit space complexity of the shortest path map is only $O(n \sqrt{n})$, while under any subset of these assumptions it is possible to construct an example of a shortest path map whose complexity is $\Omega\left(n^{2}\right)$. Note that our terrain model in Sect. 3.1 uses none of assumptions (1-3), and therefore the explicit complexity of the shortest path map in our case is $\Omega\left(n^{2}\right)$ (while our implicit construction uses only $O(n \log n)$ space).

Another well-known model is a scene where the objects are fat. There are many different definitions of fatness [1, 4, 7, 23, 25], which are all more or less equivalent. An object $f$ in $\mathbb{R}^{d}$ is $\Phi$-fat if, for any $d$-dimensional ball $B$ whose boundary intersects $f$ and whose center lies in $f, \operatorname{vol}(B \cap f) / \operatorname{vol}(B) \geq \Phi$, where $\operatorname{vol}(x)$ is the volume of $x$; a $\Phi$-fat object is called fat if $\Phi$ is some positive constant. Van der Stappen shows in [23] that a scene of pairwise openly disjoint fat objects has low density; therefore, if $P$ is a union of $O(n)$ (pairwise openly disjoint) fat tetrahedra, then, by Lemma 3.1, $P$ is uncrowded. Although we do not know how to check efficiently whether such a set of fat tetrahedra (whose union is $P$ ) exists, we overcome this difficulty in Sect. 3.4 by introducing an even broader family of polyhedra, for which our algorithm is simplest to implement. Moreover, membership in this family is easy to test if certain sufficient conditions hold (and they do hold for unions of fat tetrahedra); see Lemma 3.3 and Corollary 3.4.

\subsection{Self-conforming Polyhedra}

We say that $P$ is a self-conforming polyhedron if the following condition holds: For each edge $e$ of $\partial P$, there is a connected region $R(e)$, which is the union of $O(1)$ facets of $\partial P$ and whose interior contains $e$, so that the shortest path distance from $e$ to any edge $e^{\prime}$ of $\partial R(e)$ is at least $2 \epsilon \cdot \max \left\{|e|,\left|e^{\prime}\right|\right\}$, where $\epsilon$ is some positive constant.

If $P$ is self-conforming and if, for each edge $e^{\star}$ of $\partial P$, the region $R\left(e^{\star}\right)$ is known, then, in order to construct the conforming surface subdivision $S$, we only have to partition each edge of $\partial P$ into $E=\lceil 1 / \epsilon\rceil$ transparent edges of equal length. Each facet of $\partial P$ becomes a surface cell of $S$, bounded by $3 E$ transparent edges, and we set the well-covering region of each transparent edge $e \subseteq e^{\star}$ to be $R\left(e^{\star}\right)$. 
Fig. 14 The facet sequence $\mathcal{F}_{u}$

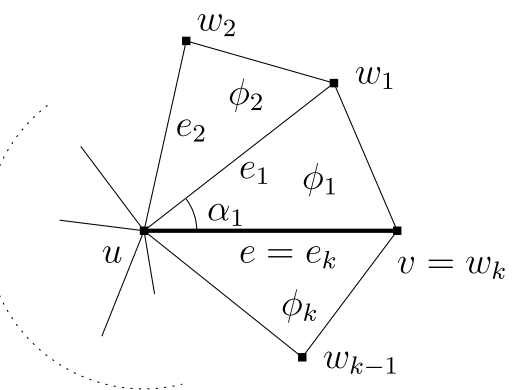

Remark Note that in this scenario the algorithm becomes much simpler, since the three-dimensional subdivision is not needed, and neither are the surface unfolding data structure and the Riemann structures. Moreover, each step of the propagation algorithm (which will be described in Sect. 4 for a more general case) is much simpler to apply within a single facet of $P$, rather than within an unfolded block tree.

Unfortunately, determining whether $P$ is self-conforming (or computing the region $R(e)$ for each edge $e$ of $\partial P$ ) may be quite cumbersome in practice. The minimal subset of facets that have to be included in $R(e)$ consists of those facets that touch $e$, which means that if $P$ is self-conforming, the maximum degree of a vertex of $P$ must be some constant $\Delta$. To find candidates for $R(e)$, we need to check all connected unions of $O(1)$ facets that contain $e$; the number of such candidates, while being constant, depends exponentially on $\Delta$.

A more practical approach is to identify easily checkable sufficient conditions for a polyhedron to be self-conforming. One such example is the class of polyhedra that satisfy the following two conditions:

(1) Each facet of $\partial P$ is fat in the plane that contains it.

(2) The maximum degree of a vertex of $P$ is $\Delta=O(1)$.

Lemma 3.3 A polyhedron that satisfies conditions (1) and (2) is self-conforming.

Proof For an edge $e=(u, v)$, denote by $R(e)$ the union of facets that are incident to $u$ or to $v$. By (2), $R(e)$ contains $O(1)$ facets. It is easy to verify that the shortest path distance from $e$ to an edge $e^{\prime}$ in $\partial R(e)$ is obtained by either a shortest path to $e^{\prime}$ from an endpoint, say $u$, of $e$, or by a shortest path to $e$ from an endpoint of $e^{\prime}$. It is also easy to see that in the former case $u$ lies on $\partial R\left(e^{\prime}\right)$; therefore, to bound the shortest path distance from $e$ to $e^{\prime}$, it suffices to consider only the shortest path distances from $e$ to the vertices of $\partial R(e)$.

Let $\mathcal{F}_{u}=\left(\phi_{1}, \ldots, \phi_{k}\right)$ be the sequence of facets that are incident to $u$, starting and ending with a facet bounded by $e$ (there are in fact two such possible sequences, each being the reverse of the other), and denote by $\left(w_{1}, \ldots, w_{k}\right)$ the sequence of their vertices that are distinct from $u$, so that $w_{i} \in \phi_{i}$ and $w_{k}=v$. For each $1 \leq i \leq k$, denote by $e_{i}$ the edge $\left(u, w_{i}\right)$ and denote by $\alpha_{i}$ the angle of $\phi_{i}$ at $u$ (see Fig. 14). Denote by $\mathcal{F}_{u}(i, j)$ the subsequence $\left(\phi_{i}, \ldots, \phi_{j}\right)$ of $\mathcal{F}_{u}$ and denote by $\alpha(i, j)$ the $\operatorname{sum} \alpha_{i}+\cdots+\alpha_{j}$ for each $1 \leq i \leq j \leq k$. 
Fig. 15 If

$\alpha=\alpha(1, i) \leq \alpha(i+1, k)<\pi / 2$,

the shortest path distance

$d\left(w_{i}, e\right)$ cannot be smaller

than $H$. (The unfolded facets of

$\mathcal{F}_{u}(1, i)$ are shaded $)$

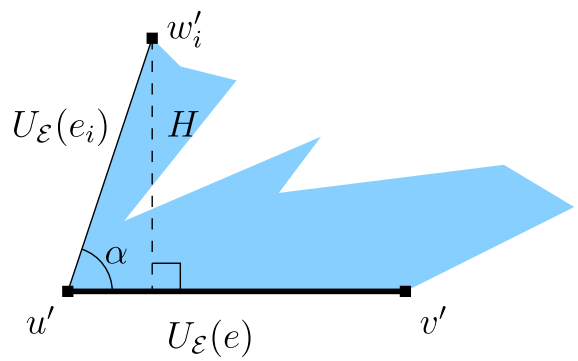

Fix an index $1 \leq i \leq k$. Assume first that $\min \{\alpha(1, i), \alpha(i+1, k)\} \geq \pi / 2$. Then the shortest path distance $d\left(w_{i}, e\right)$ is $\left|e_{i}\right|$. (Indeed, any other path from $w_{i}$ to $e$ has to traverse one of the sequences $\mathcal{F}_{u}(1, i), \mathcal{F}_{u}(i+1, k)$ and, within these unfolded sequences, $u$ is the point on $e$ nearest to $w_{i}$.) Since each facet in $\mathcal{F}_{u}(1, i)$ is fat, $|e|$ is at most $O(1)$ times greater than $\left|e_{1}\right|$, which is at most $O(1)$ times greater than $\left|e_{2}\right|$, and so on; since $i \leq k \leq \Delta=O(1),|e|$ is at most $O(1)$ times greater than $\left|e_{i}\right|$.

Suppose next that $\min \{\alpha(1, i), \alpha(i+1, k)\}<\pi / 2$; without loss of generality, assume that $\alpha(1, i) \leq \alpha(i+1, k)$. Denote by $\mathcal{E}=\left(e_{1}, \ldots, e_{i-1}\right)$ the corresponding edge sequence of $\mathcal{F}_{u}(1, i)$ and denote by $x^{\prime}$ the unfolded image $U_{\mathcal{E}}(x)$ for any point $x$ in a facet of $\mathcal{F}_{u}(1, i)$. The shortest path distance $d\left(w_{i}, e\right)$ cannot be smaller than the height $H$ of the unfolded triangle $\Delta u^{\prime} w_{i}^{\prime} v^{\prime}$, subtended from $w_{i}^{\prime}=U_{\mathcal{E}}\left(w_{i}\right)$ to the base $u^{\prime} v^{\prime}=U_{\mathcal{E}}(e)$ (see Fig. 15). It is not difficult to see that, since (i) each facet in $\mathcal{F}_{u}(1, i)$ is fat, (ii) $\mathcal{F}_{u}(1, i)$ contains only $O(1)$ (triangular) facets, and (iii) $\alpha(1, i)<\pi / 2$, $\Delta u^{\prime} w_{i}^{\prime} v^{\prime}$ is also fat (although the bound on its fatness depends on $i$ ). This follows because, as argued above, $\left|u^{\prime} v^{\prime}\right|=|e|$ is at most $O(1)$ times greater than $\left|u^{\prime} w_{i}^{\prime}\right|=\left|e_{i}\right|$ and because $\alpha(1, i)$ must be at least some fixed constant value (as follows from the fatness of the facets in $\left.\mathcal{F}_{u}(1, i)\right)$. Therefore, $H$ is at most $O(1)$ times smaller than $\left|u^{\prime} v^{\prime}\right|=|e|$.

Hence, in either case, there is some positive constant $c_{1}$ such that $d\left(w_{i}, e\right) \geq c_{1}|e|$ for each $i$; in particular, we also have $d\left(w_{i-1}, e\right) \geq c_{1}|e|$.

Since each facet in $\mathcal{F}_{u}$ is fat, the distance from $u$ to the opposite edge $e_{i}^{\prime}=w_{i-1} w_{i}$ of $\phi_{i}$ for each $\phi_{i} \in \mathcal{F}_{u}$ is at most $O(1)$ times smaller than $\left|e_{i}\right|$ and therefore at most $O(1)$ times smaller than $|e|$. Hence, there is some positive constant $c_{2}$ such that $d\left(u, e_{i}^{\prime}\right) \geq c_{2}|e|$. Similarly, there is some positive constant $c_{3}$ such that $d\left(v, e_{i}^{\prime}\right) \geq$ $c_{3}|e|$.

We can therefore conclude that there is a positive constant $c=\min \left\{c_{1}, c_{2}, c_{3}\right\}$ for which $d\left(e, e_{i}^{\prime}\right) \geq c|e|$, and we can repeat all the above arguments for $e_{i}^{\prime}$ instead of $e$ to show that there is another positive constant $c^{\prime}$ such that $d\left(e, e_{i}^{\prime}\right) \geq c^{\prime}\left|e_{i}^{\prime}\right|$. In summary, we have

$$
d\left(e, e_{i}^{\prime}\right) \geq \min \left\{c, c^{\prime}\right\} \max \left\{|e|,\left|e_{i}^{\prime}\right|\right\},
$$

and this completes the proof of the lemma.

Corollary 3.4 A polyhedral complex $P$ that is the union of $O(n)$ pairwise openly disjoint fat tetrahedra (where $n$ is the number of vertices of $\partial P$ ) is self-conforming.

Proof Clearly, $P$ satisfies conditions (1) and (2). 
Fig. 16 (a) The unfolded angle $\angle w u v \geq \pi / 2$, and therefore $d(w, e)=\left|e^{\prime}\right|$. (b) The unfolded angle $\angle w u v<\pi / 2$, and therefore $d(w, e)$ is at least the height in $\Delta u w v$ subtended from $w$ to the base $u v$

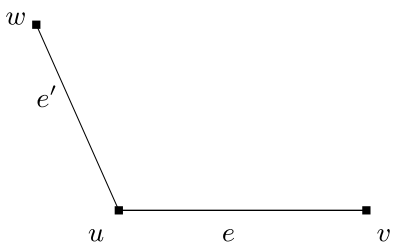

(a)

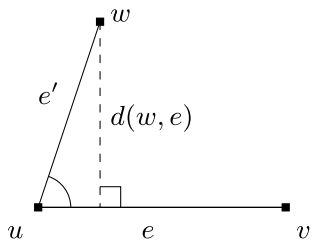

(b)

Analysis of the Geometric Parameters Since our algorithm for a polyhedron $P$ that satisfies conditions (1) and (2) seems simple enough to be practical, we provide a more careful analysis of the geometric parameters that affect the time and space complexity of the algorithm.

It follows from the proof of Lemma 3.3 that the number of resulting transparent edges for a polyhedron $P$ that satisfies the conditions (1) and (2) is $O(n E)$ (that is, each edge of $P$ is partitioned into $E$ transparent edges) and that $E=$ $2\left\lceil\max _{e}\{|e| / d(e)\}\right\rceil$, where $d(e)$ is the minimum shortest path distance from the edge $e$ to the other endpoint of an edge of $P$ which shares one endpoint with $e$. In the following lemma, we bound $|e| / d(e)$ by using a pair of additional, easily computable geometric parameters: $\Lambda$, the maximum length ratio between a pair of edges sharing a common endpoint, and $\beta_{\mathrm{min}}$, the minimum angle of a facet of $\partial P$. (If the maximum fatness parameter of the facets of $P$ is $\Phi$, we have $\beta_{\min } /(2 \pi) \geq \Phi$ and therefore $\beta_{\min } \geq 2 \pi \Phi$.)

Lemma 3.5 For a polyhedron $P$ that satisfies conditions (1) and (2), the conforming surface subdivision $S$ can be constructed by partitioning each edge of $P$ into $O\left(\Lambda / \sin \beta_{\min }\right)$ transparent edges (of equal length).

Proof As stated above, each edge of $P$ is subdivided into $2\left\lceil\max _{e}\{|e| / d(e)\}\right\rceil$ transparent edges, where $d(e)$ is the minimum shortest path distance from the edge $e$ to the other endpoint of an edge of $P$ which shares one endpoint with $e$.

Let $e=(u, v)$ be an edge of $P$ for which $|e| / d(e)$ is maximal, and let $w$ be the vertex of $P$ for which $d(w, e)=d(e)$. Without loss of generality, assume that $w$ is connected to $u$ by the edge $e^{\prime}=(u, w)$ of $P$. See Fig. 16 for an illustration.

By the proof of Lemma 3.3, either (i) $d(w, e)=\left|e^{\prime}\right|$, and therefore $|e| / d(e) \leq \Lambda$, or (ii) $d(w, e)$ is at least the height in the (unfolded) triangle $\Delta u w v$ subtended from $w$ to the base $u v$, and $\beta_{\min } \leq \angle w u v<\pi / 2$; since $d(w, e)=\left|e^{\prime}\right| \sin \angle w u v$, we have $|e| / d(e) \leq \Lambda / \sin \beta_{\min }$, and the lemma follows.

Remark In Lemma 3.5, we have used the global parameters $\Lambda$ and $\beta_{\min }$ to subdivide each edge $e$ of $P$. It is plausible that the conforming surface subdivision $S$ can be constructed by subdividing each edge $e$ of $P$ into a number of transparent edges that depends only on local values $\Lambda(e), \beta_{\min }(e)$, computed over a "small" number of facets around each $e$. Obviously, for many "real-life" polyhedra, such an optimization could greatly reduce the number of transparent edges in $S$, improving the time and space complexity of the algorithm. We leave this question open for future research and/or experimenting. 


\section{Wavefront Propagation}

In this section we complete the presentation of the algorithm by describing its main phase, wavefront propagation. As already noted, the changes that are required to handle the nonconvex models are more minor here. All these changes are caused by the fact that a shortest path may pass through one or several s-vertices.

Wavefront Propagation on a Convex Polytope: A review We begin with a brief review of the wavefront propagation algorithm on the surface of a convex polytope [19]; then we will describe the required adjustments for the nonconvex cases. The continuous Dijkstra paradigm simulates a unit-speed wavefront expanding from the given source point $s$, and spreading along the surface of the polytope.

At simulation time $t$, the true wavefront $W_{t}$ consists of points whose shortest path distance to $s$ along $\partial P$ is $t$; we say that all such paths are encoded in $W_{t}$. A wave $w$ is a continuous section of $W_{t}$ whose unfolded image is a circular arc (on any plane of unfolding); its center $s^{\prime}$ is an unfolded image of $s$ (that is, a source image), and it is called the generator of $w$. Note that, given $w$ (at a fixed time $t$ ) and an unfolded sequence $\mathcal{F}_{w}$ of facets whose union contains $w, s^{\prime}$ is unique, and so is the edge sequence $\mathcal{E}$ for which $U_{\mathcal{E}}(s)=s^{\prime}$. (When $\mathcal{F}_{w}$ and, consequently, the plane of unfolding are fixed, we say that $\mathcal{E}$ is the edge sequence of $s^{\prime}$ at $t$.) Note that $\mathcal{E}$ does not include the edges that are incident to $s$, since none of them is intersected by a shortest path encoded in $w$. See [19] for more details concerning edge sequences.

As the simulation time $t$ increases, the meeting points of the wave $w_{i}$ generated by $s_{i}$ with each of its (at most two) adjacent waves trace two bisectors, each of which is the locus of points equidistant from the generators of the two corresponding waves. The bisector of the two consecutive generators $s_{i}, s_{i+1}$ in the wavefront is denoted by $b\left(s_{i}, s_{i+1}\right)$, and its unfolded image is a straight line. We say that $s_{i}$ claims every point that lies closer to $s_{i}$ than to its neighbors $s_{i-1}, s_{i+1}$ in the wavefront; note that all such points lie between $b\left(s_{i}, s_{i-1}\right)$ and $b\left(s_{i}, s_{i+1}\right)$.

We say that a transparent edge $e$ is covered by $W_{t}$ when each point in $e$ is crossed by a shortest path encoded in $W_{t}$ (at simulation time $t$ or earlier). When $e$ is covered by $W_{t}$, there exists a pairwise openly disjoint decomposition of $e$ into nonempty intervals, each of which is claimed by a different generator of $W_{t}$. We simulate this decomposition of $e$ (by the true wavefront $W_{t}$ ) by two one-sided wavefronts, each of which reaches $e$ from a distinct fixed side, so that, for any point $p \in e$, the true distance $d(s, p)$ is the minimum of the lengths of the two paths from $s$ to $p$ that are encoded in the one-sided wavefronts for the opposite sides of $e$. See Fig. 17.

For a transparent edge $e$, a one-sided wavefront $W(e)$ is represented as a sequence of the source images that generate the waves of $W(e)$, all unfolded to a common plane. The wavefront is stored in a persistent binary tree structure that combined properties of the data structure of Mount [16] with those of [9].

We maintain a timer that controls the wavefront propagation simulation; when it reaches a conservative (but not too conservative) upper bound covertime(e) on the time when a transparent edge $e$ is covered by the wavefront, the continuous Dijkstra algorithm processes $e$ : We construct each of the one-sided wavefronts at $e$ by merging 
Fig. 17 Some of the waves of the two one-sided wavefronts $W(e)$ and $W^{\prime}(e)$ are absent from the true wavefront, since there is another wave in the opposite one-sided wavefront that reaches the same points of $e$ (before they do)

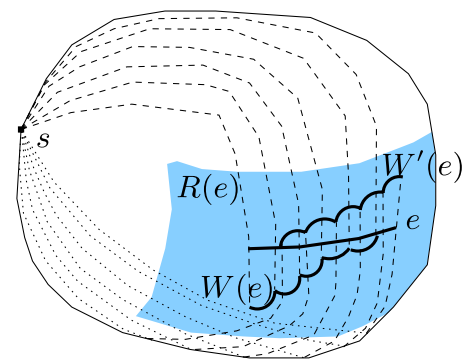

the wavefronts that have already reached $e$ from the respective sides, and propagate each of these one-sided wavefronts further into the cell on the other side of $e$. The transparent edges whose one-sided wavefronts were propagated to be merged at $e$ are the $O(1)$ edges on the boundary of the well-covering region $R(e)$, denoted as input $(e)$; the transparent edges that the one-sided wavefronts of $e$ were propagated to are the set output $(e)=\{f \mid e \in \operatorname{input}(f)\}$ (and it is shown in [19] that $\mid$ output $(e) \mid=$ $O(1)$ for each $e$ ). The following important property of the simulation timer, which is proved in [19], allows each transparent edge to be processed exactly once by the continuous Dijkstra algorithm.

Lemma 4.1 (Consistent Synchronization Lemma) If there exists a path $\pi$ from $s$ that reaches a transparent edge e before time covertime(e), so that a prefix of $\pi$ belongs to a one-sided wavefront at a transparent edge $f \in \operatorname{input}(e)$, then covertime $(f)<$ covertime $(e)$.

The soundness of the merging and the propagation steps, ascertained in [19] (by an inductive proof, in Lemmas 4.5 and 5.11 of [19]), completes the correctness analysis of the algorithm.

The actual procedure of propagating a one-sided wavefront $W(e)$ from $e$ to output(e) is implemented as a constant number of steps, in each of which the wavefront is propagated through a single surface cell, until each path in $W(e)$ reaches edges in output(e) (or is eliminated if the algorithm detects that it is not a shortest path).

Each propagation step of a one-sided wavefront $W(e)$ from a transparent edge $e$ through a surface cell $c$ that is bounded by $e$ is implemented by propagating $W(e)$ through the block trees of the Riemann structure $\mathcal{T}(e)$. Each sub-wavefront of $W(e)$ that follows a different path in a block tree is called topologically constrained, since the block sequence that it traverses corresponds to a fixed homotopy class of paths from $e$ to another transparent edge of $\partial c$ (there are $O(1)$ such homotopy classes, since $c$ may contain at most one vertex of $P$ and at most $O(1)$ holes). During each such propagation, we keep track of combinatorial changes that occur within the wavefront: at each such event, we either split a wave when it hits a vertex of $P$ (recall that, in the convex case, shortest paths cannot go through vertices of $P$ ) or eliminate a wave when it is "overtaken" by its two neighbors; see Fig. 18. Interactions between waves of different (topologically constrained) wavefronts are partially ignored. They come into effect when we merge wavefronts that reach some transparent edge, see [19] and below. 


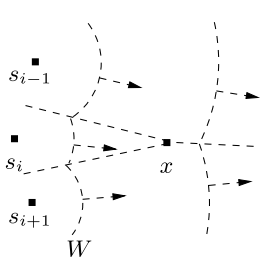

(a)

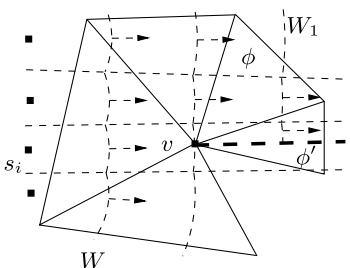

(b)

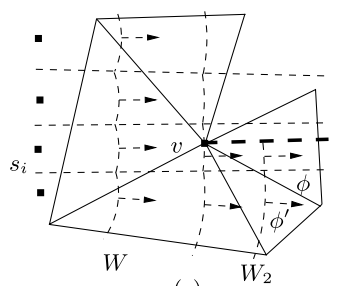

(c)

Fig. 18 (a) The wavefront $W$ before and after the bisector event at $x$, where the wave of the generator $s_{i}$ is eliminated from $W$. (b), (c) Vertex event: splitting a wavefront $W$ at a vertex $v$ into two new wavefronts $W_{1}, W_{2}$, which are propagated separately beyond $v$ through different unfoldings of the facet sequence around $v$-note that the images of the facets $\phi, \phi^{\prime}$ in (b) are different from those in (c)

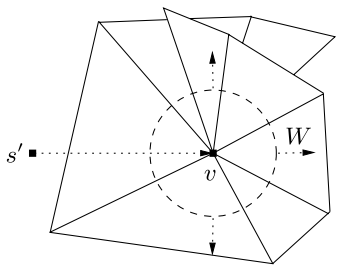

(a)

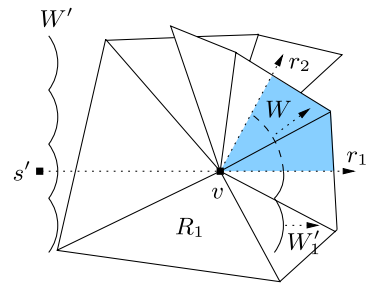

(b)

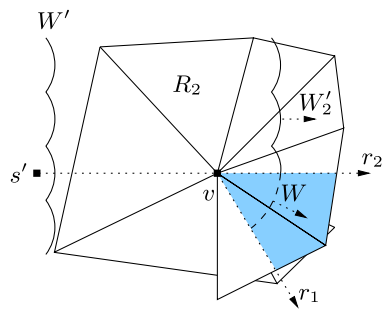

(c)

Fig. 19 (a) When an s-vertex $v$ is reached by the wavefront, a new singleton wavefront $W=(v)$ is created. (b) (resp., (c)) Each point in the region $R_{1}$ below the ray $r_{1}$ (resp., $R_{2}$ above the ray $r_{2}$ ) which emanates from $s^{\prime}$ through $v$ in the corresponding unfolded Riemann layer is reached by $W_{1}^{\prime}$ (resp., $W_{2}^{\prime}$ ) before it is reached by $W$, and therefore $W$ can be trimmed to lie only between $r_{1}$ and $r_{2}$. For each point $p$ in the shaded region, the path $\pi\left(s^{\prime}, p\right)$ encoded in $W$ is necessarily shorter than any path encoded in $W_{1}^{\prime}$ or $W_{2}^{\prime}$

Adjustments of the Algorithm for the Nonconvex Cases The wavefront propagation algorithm for a nonconvex $P$ requires several modifications from the version for the convex case, as studied in [19], all caused by the fact that a shortest path may pass through one or several s-vertices.

When an s-vertex $v$ is reached by (a wave from) a generator $s^{\prime}$ in a wavefront $W^{\prime}$ at time $t_{v}=d\left(s^{\prime}, v\right)$, two things happen. First, $W^{\prime}$ is split into a pair of subwavefronts $W_{1}^{\prime}, W_{2}^{\prime}$, which "bypass" $v$ on two different sides, as in the convex case. Second, unlike the convex case, a new singleton wavefront $W=(v)$ is created, where its single wave expands from $v$ across each of the facets adjacent to $v$; see Fig. 19(a). At any time $t \geq t_{v}, W$ is the locus of endpoints of all the shortest paths of length $t-t_{v}$ from $v$.

Of course, the actual portion of $W$ that encodes shortest paths (from $s$ ) is not the whole (cyclic) $W$, since there are points that are reached faster by paths that do not pass through $v$. In particular, denote by $R_{1}$ and $R_{2}$ the respective regions of $\partial P$ into which $W_{1}^{\prime}, W_{2}^{\prime}$ are propagated (bounded by the two continuations $r_{1}, r_{2}$ of the ray from $s^{\prime}$ through $v$, on the respective Riemann layers of the unfolded surface of $P$ ), including the respective portions reached by $W_{1}^{\prime}, W_{2}^{\prime}$ up to the time they encounter $v$ (as part of $W^{\prime}$ ), see Fig. $19(\mathrm{~b}-\mathrm{c})$. All points in $R_{1}$ and $R_{2}$ can be reached faster 
by shortest paths encoded in $W_{1}^{\prime}$ or $W_{2}^{\prime}$, respectively, than by paths encoded in $W$, as follows easily from the triangle inequality. We could have, therefore, right away mark as irrelevant the portion of $W$ that is propagated into $R_{1}$ or $R_{2}$. In fact, the remaining portion of $W$ (bounded by $r_{1}$ and $r_{2}$ ) can be considered as a third sub-wavefront which enters the split $W^{\prime}$ and is positioned between $W_{1}^{\prime}$ and $W_{2}^{\prime}$. Nevertheless, to simplify the algorithm, we do not prune $W$ nor position it between $W_{1}^{\prime}$ and $W_{2}^{\prime}$. Instead, we propagate it independently, until it is merged with other (topologically constrained) wavefronts, including $W_{1}^{\prime}, W_{2}^{\prime}$, as described below.

As the simulation time $t$ evolves, $W$ may split into several waves, which behave similarly to waves generated by $s$ - each of them is a continuous section of $W$ whose unfolded image is a circular arc (on any plane of unfolding), and its generator (the center of the arc) is an unfolded image of $v$. Each such generator $s^{\prime}$ is assigned an additive weight $\delta\left(s^{\prime}\right)=d\left(s^{\prime}, v\right)$, and the generator that has claimed $v$ is recorded as the predecessor of $v$. (The weight of the images of $s$ is 0 , and only the images of $s$ have no predecessors.)

The high-level description of the continuous Dijkstra algorithm for a nonconvex $P$ is similar to the case where $P$ is convex. In particular, the wavefront $W$ generated at an s-vertex $v$ contributes to the merging process at each transparent edge (which it reaches) that bounds any well-covering region $R$ that contains $v$, as does any other wavefront that enters $R$. However, the implementation details for the nonconvex case are somewhat more involved, as described next.

First, the unfolded image of the bisector $b\left(s^{\prime}, s^{\prime \prime}\right)$ of a pair of generators $s^{\prime}, s^{\prime \prime}$ is a branch of a hyperbola (which can degenerate into a straight line when $s^{\prime}, s^{\prime \prime}$ have the same weight $){ }^{7}$ so that for each point $p \in b\left(s^{\prime}, s^{\prime \prime}\right)$, we have $\delta\left(s^{\prime}\right)+d\left(s^{\prime}, p\right)=$ $\delta\left(s^{\prime \prime}\right)+d\left(s^{\prime \prime}, p\right)$.

Therefore, $b\left(s^{\prime}, s^{\prime \prime}\right)$ can intersect a transparent edge in (at most) two points (rather than in at most one point in the case where $P$ is convex and each bisector is unfolded into a straight line). This could potentially damage the merging procedure at a transparent edge $e$. In this step, we determine which generators of the wavefronts that have reached $e$ are absent from the resulting merged one-sided wavefront $W(e)$, and we do it by analyzing the order along $e$ of the intersection points of $e$ with the bisectors between the tested generators; see [19] for a detailed description of this procedure. The danger is that this order may be undefined when a bisector intersects $e$ twice. However, as the following lemma shows, since we merge only wavefronts that reach $e$ from a common side, this situation cannot arise. Specifically, we have: ${ }^{8}$

Lemma 4.2 Let $s^{\prime}, s^{\prime \prime}$ be a pair of generators that participate in the merging procedure at a transparent edge $e$ when a one-sided wavefront $W(e)$ from a fixed side of $e$ is being computed. Then the bisector $b\left(s^{\prime}, s^{\prime \prime}\right)$ intersects $e$ in at most one delimiter

\footnotetext{
${ }^{7}$ As mentioned above, assuming general position, this will happen only when $s^{\prime}, s^{\prime \prime}$ are both images of the same s-vertex.

${ }^{8}$ Note that Lemma 4.2 is similar to a result from [9], in which hyperbolae are ubiquitous; however, the proofs are somewhat different.
} 
Fig. 20 The dotted lines are the asymptotes of the hyperbola that contains $b\left(s^{\prime}, s^{\prime \prime}\right)$; it is easy to see that no straight line that intersects $b\left(s^{\prime}, s^{\prime \prime}\right)$ twice can intersect $\ell$ to the left of $s^{\prime \prime}$ (or, more strongly, to the left of the center $o$ of the hyperbola)

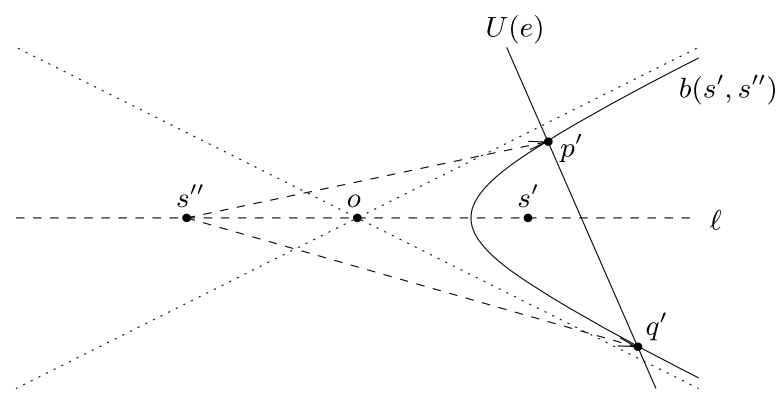

point of the subdivision of $e$ into portions claimed by the generators of $W(e)$ (and therefore in at most one point in $\operatorname{SPM}(s))$.

Proof Assume to the contrary that $b\left(s^{\prime}, s^{\prime \prime}\right)$ intersects $e$ in two delimiter points $p, q$ of the subdivision of $e$ into portions claimed by the generators of $W(e)$. Denote by $p^{\prime}, q^{\prime}$ the respective images of these points unfolded onto the same plane as $s^{\prime}, s^{\prime \prime}$, and $e$ (on which the merging procedure takes place).

Since $b\left(s^{\prime}, s^{\prime \prime}\right)$ is a branch of a hyperbola whose major axis is the line $\ell$ through the foci $s^{\prime}, s^{\prime \prime}$, it is easy to check that $p^{\prime}$ and $q^{\prime}$ must lie on different sides of $\ell$. Indeed, if they lied on the same side of $\ell$, then the line through them (which contains the unfolded image of $e$ ) would separate $s^{\prime}$ and $s^{\prime \prime}$, contrary to the assumption that $s^{\prime}$ and $s^{\prime \prime}$ belong to wavefronts that reach $e$ from the same side. Moreover, in this setup, exactly one of them must lie in the interior of the triangle formed by $p^{\prime}, q^{\prime}$, and the second generator; without loss of generality, assume that $s^{\prime}$ lies in the interior of $\Delta p^{\prime} q^{\prime} s^{\prime \prime}$, see Fig. 20.

However, since $s^{\prime \prime}$ claims (together with $s^{\prime}$ ) the points $p$ and $q$ in $W(e)$, the portion of $\partial P$ whose unfolded image is $\Delta p^{\prime} q^{\prime} s^{\prime \prime}$ is a simply connected region that is part of a single facet sequence $\mathcal{F}$ between a facet incident to (the vertex whose image is) $s^{\prime \prime}$ and a facet intersected by $e$ (that is, the corresponding edge sequence of $\mathcal{F}$ is the edge sequence of $s^{\prime \prime}$ at the simulation time covertime $\left.(e)\right)$. Therefore, the interior of $\Delta p^{\prime} q^{\prime} s^{\prime \prime}$ cannot contain $s^{\prime}$, since it is an image of a vertex ( $s$ or another s-vertex), a contradiction.

Remark The proof of Lemma 4.2 (particularly, the fact that the situation depicted in Fig. 20 is impossible) has another important implication. Consider a pair of generators $s^{\prime}, s^{\prime \prime}$ that are part of a common one-sided wavefront $W(e)$ at some transparent edge $e$, or, more generally, that take part in the merging process that creates $W(e)$. Then the "relevant" portion of the bisector $b\left(s^{\prime}, s^{\prime \prime}\right)$ that can be actually traced by the respective waves of $s^{\prime}, s^{\prime \prime}$ in the wavefront lies on one side of the straight line through $s^{\prime}, s^{\prime \prime}$ (which is the major axis of the hyperbola that contains $b\left(s^{\prime}, s^{\prime \prime}\right)$ ). This, in turn, implies that the distances $d\left(s^{\prime}, p\right), d\left(s^{\prime \prime}, p\right)$ grow as the point $p$ slides along the "relevant" portion of $b\left(s^{\prime}, s^{\prime \prime}\right)$; this property is used by our wavefront propagation algorithm in the convex case and, consequently, also in the current extension. (See the correctness analysis in [19] for further details; the analysis is essentially identical for the current scenario.) 
Fig. 21 The point $p^{\prime}$ lies within the shaded triangle formed by $\pi\left(s_{i-1}, p\right), \pi\left(s_{i}, p\right)$, and $e$

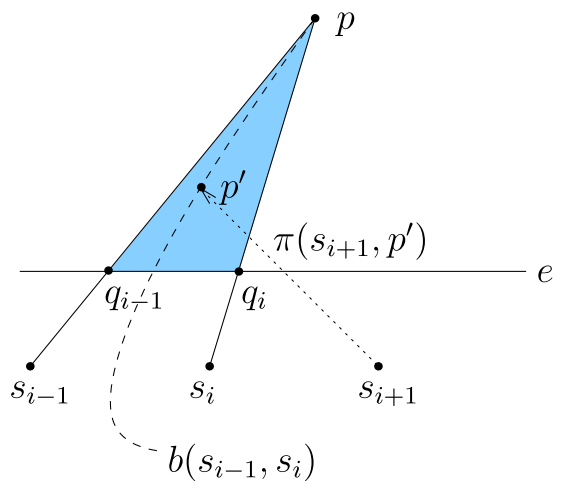

Another problem, similar to the one solved by Lemma 4.2, is that a pair of (hyperbolic) bisectors can intersect each other in up to four points (rather than only one point in the convex case), which could have complicated the computation of bisector event locations. However, the following lemma shows that this problem does not arise in the algorithm.

Lemma 4.3 Let $s_{i-1}, s_{i}, s_{i+1}$ be consecutive generators in a (topologically constrained) one-sided wavefront $W(e)$ at a transparent edge e. If the bisectors $b\left(s_{i-1}, s_{i}\right)$ and $b\left(s_{i}, s_{i+1}\right)$ intersect each other beyond $e$, then there is exactly one such intersection point.

Proof Assume to the contrary that there are at least two such intersection points $p, p^{\prime} \in b\left(s_{i-1}, s_{i}\right) \cap b\left(s_{i}, s_{i+1}\right)$ beyond $e$ and assume, without loss of generality, that $p^{\prime}$ precedes $p$ along $b\left(s_{i-1}, s_{i}\right)$ (that is, $p^{\prime}$ is closer to each of $s_{i-1}, s_{i}$ than $p$ ). Denote by $q_{i-1}, q_{i}$ the respective intersection points of $\pi\left(s_{i-1}, p\right), \pi\left(s_{i}, p\right)$ with $e$, see Fig. 21 for an illustration.

The portion of $b\left(s_{i-1}, s_{i}\right)$ between $e$ and $p$ is contained in the triangle $\Delta q_{i-1} p q_{i}$, and therefore the path $\pi\left(s_{i+1}, p^{\prime}\right)$ must either intersect the portion of $e$ between $q_{i-1}$ and $q_{i}$, which contradicts the fact that $s_{i-1}, s_{i}, s_{i+1}$ are consecutive generators in $W(e)$, or intersect one $\pi\left(s_{i}, p\right)$ or $\pi\left(s_{i-1}, p\right)$. Since $\pi\left(s_{i+1}, p^{\prime}\right), \pi\left(s_{i}, p\right)$, and $\pi\left(s_{i-1}, p\right)$ are all shortest paths from $s$ (in the modified environment where no other generators reach $\Delta q_{i-1} p q_{i}$ ), they cannot cross each other, a contradiction.

Another difference from the convex case is as follows. Propagating a one-sided wavefront $W(e)$ through a cell $c$ that contains an s-vertex $v$ has to be modified to handle paths through $v$. To do so, we also propagate the singleton wavefront $W(v)=(v)$ through the Riemann structure $\mathcal{T}(v)$, as described in Sect. 3.1.

To compute $\delta(v)$, we proceed as follows. At the beginning of the continuous Dijkstra algorithm, we initialize weight $(v):=+\infty$ for each s-vertex $v$. Whenever a wavefront $W$ that is propagated though $c$ reaches $v$, we update weight $(v):=$ $\min \left\{\right.$ weight $\left.(v), \delta\left(s_{i}\right)+d\left(s_{i}, v\right)\right\}$, where $s_{i} \in W$ is the generator that claims $v$ (among all the generators in $W) ; \delta(v)$ is the final value of weight $(v)$. Note that the value of weight $(v)$ does not affect the shortest paths from $v$ to the transparent edges of $\partial c$. In 
principle, we could compute the (topologically constrained) wavefronts $W(v, e)$ for each transparent edge $e \in \partial c$ without knowing $\delta(v)$, even ahead of time. However, to merge these wavefronts with other wavefronts that reach the same transparent edges, we need to know $\delta(v)$.

Lemma 4.4 If an $s$-vertex $v \in R(e)$ is a generator in one of the wavefronts that reach a transparent edge e before the time covertime (e) (in which the merging process at $e$ takes place), then the current value of weight $(v)$ is final (and is equal to $\delta(v)$ ).

Proof We add the assumption of the lemma to the inductive assertion about the correctness of the propagation algorithm, as given in [19]. Among other properties, this assertion states that, for each edge $f$, at simulation time covertime $(f)$, the following holds. First, both one-sided wavefronts at $f$ have been correctly created (that is, together they encode all the shortest paths from $s$ to points of $f$ ). Second, each such one-sided wavefront $W(f)$ has been correctly propagated toward the edges of output $(f)$ through the appropriate block trees; that is, each bisector or vertex event on the way of $W(f)$ from $f$ to output $(f)$ has been correctly processed.

Assume now that the inductive properties hold for all transparent edges $f$ with covertime $(f)<$ covertime $(e)$ and suppose to the contrary that $v$ is reached by a path $\pi$ from $s$ so that $|\pi|<\operatorname{weight}(v)$, where $\operatorname{weight}(v)$ is the weight of $v$ at the time of the merging process at $e$. Since there can be no other s-vertex in $R(e)$ except $v$ (including $s), \pi$ must cross $\partial R(e)$; denote by $f$ the transparent edge through which $\pi$ enters $R(e)$ (for the last time).

If covertime $(f)<$ covertime $(e)$, then, by the inductive assumption, the one-sided wavefronts at $f$ have been correctly created at time covertime $(f)$, and therefore the prefix of $\pi$ up to $f$ is encoded in the relevant one-sided wavefront $W(f)$. Moreover, $W(f)$ has been correctly propagated, at simulation time covertime $(f)$, toward the edges of output $(f)$ through the appropriate block trees, and the corresponding vertex event at $v$ is therefore detected and correctly processed by the algorithm. At this event we would have updated $\delta(v):=|\pi|$; this, however, contradicts the fact that weight $(v)>|\pi|$ at simulation time covertime $(e)>\operatorname{covertime}(f)$.

Otherwise, we can omit the case where covertime $(e)=\operatorname{covertime}(f)$ by assuming general position. If covertime $(e)<\operatorname{covertime}(f)$, consider a path $\pi^{\prime}$ through $v$ that reaches $e$ before time covertime $(e)$, and denote its portion between $v$ and $e$ by $\pi(v, e)$ (that is, $\left.\left|\pi^{\prime}\right|=\operatorname{weight}(v)+|\pi(v, e)|\right)$. Since $|\pi|<\operatorname{weight}(v)$, there is a path $\tilde{\pi}=$ $\pi \| \pi(v, e)$ through $f$ to $e$ that reaches $e$ before time covertime $(e)$. That is, there exists a path $\tilde{\pi}$ that reaches $e$ before time covertime $(e)$, so that a prefix of $\tilde{\pi}$ is encoded in a one-sided wavefront at the edge $f \in \operatorname{input}(e)$, which implies, by Lemma 4.1, that covertime $(f)<\operatorname{covertime}(e)$, a contradiction.

One can easily check that the remaining details of the wavefront propagation algorithm for the convex case can be applied, essentially verbatim, in the cases of the realistic nonconvex polyhedra described in this paper.

After the wavefront propagation phase, shortest path queries can be answered similarly to the way they are answered in [19] with the following nuance. In the case of an uncrowded polyhedron, and in the case of a terrain with bounded facet slopes, we 
can find the surface cell that contains the query point $q$, using the three-dimen sional subdivision $S_{3 D}$ preprocessed for point location as in [19]. However, this cannot be done in the case of a self-conforming polyhedron (since we do not construct $S_{3 D}$ there). We therefore assume that, for self-conforming polyhedra, the facet of $\partial P$ that contains $q$ is either given or can be computed by some other efficient procedure.

Theorem 4.5 (Main Result) Let $P$ be a polyhedron with $n$ vertices that is either (i) self-conforming, or (ii) uncrowded, or (iii) a terrain whose maximum facet slope is a constant. Given a source point $s \in \partial P$, we can construct an implicit representation of the shortest path map from $s$ on $\partial P$ in $O(n \log n)$ time and space. Using this structure, we can identify, and compute the length of, the shortest path from $s$ to any point $q \in \partial P$ in $O(\log n)$ time in the real RAM model (provided that, in case (i), the facet of $\partial P$ that contains $q$ is also given or computable in $O(\log n)$ time). A shortest path $\pi(s, q)$ can be computed in additional $O(k)$ time, where $k$ is the number of straight edges in the path.

\section{Extensions and Remarks}

We have extended our optimal-time algorithm for shortest paths on a convex polytope [19], making it also applicable for several classes of realistic nonconvex polyhedra. Concerning these classes, we note that a terrain with a bounded maximum facet slope is a very natural and useful model; the uncrowded polyhedra fit well into the hierarchy of realistic models discussed in [6,7]; a self-conforming polyhedron is a more restrictive but still natural and realistic model, and it allows for a major simplification of the algorithm. We have also shown that the data structure of Mount [16] (which we have already extended in [19] to a "kinetically evolving" version that can be constructed in $O(n \log n)$ time) can easily be extended for nonconvex polyhedra, and its construction time remains optimal, for the models discussed in this paper.

As in the cases studied in [9, 19], the described solution can also easily be extended to the case of multiple sources, which is equivalent to computing their (implicit) geodesic Voronoi diagram, a partition of $\partial P$ into regions so that all points in a region have the same nearest source and the same combinatorial structure of the shortest path from that source. Here, given a query point $q \in \partial P$, we wish to return the shortest path length (and, possibly, the shortest path itself) from $q$ to the nearest source point or, simply, to identify this source point. Another easy extension is where each source can have its specified release time, at which it starts being active, which can be encoded by increasing the additive weight of the corresponding generator. In both cases, as in [19], the time and space complexity of the algorithm is $O((m+n) \log (m+n))$, where $m$ is the number of sources.

\section{Open Problems}

1. The fact that our algorithms for each of the discussed models depend mainly on the existence of a conforming surface subdivision suggests that there might be a higher-level theorem that encompasses these special cases, giving natural sufficient conditions for the existence of such a subdivision. 
2. How realistic are the models considered in this paper? The measurements of the density and the clutter factors in [7] suggest that our crowdedness parameter may be low for many real-world inputs. It is also of interest to determine how common are the self-conforming polyhedra, due to the (relative) simplicity of the algorithm in this case.

3. The upper bounds on the constants that are hidden in the time complexity of the propagation algorithm in [19] and, consequently, of the algorithm in the current paper, for the uncrowded polyhedra and for the terrain model, are quite large. An optimization is needed before the algorithm for these models can be implemented to run efficiently in practice (the situation is much better for self-conforming polyhedra).

4. Since our algorithm is relatively simple for the self-conforming scenario, it is interesting to find additional families of polyhedra that can be easily (and efficiently) tested for being self-conforming.

5. In the self-conforming scenario, we have used global geometric parameters to subdivide each edge $e$ of $P$; it is plausible that local parameters (which consider only "a few" facets around $e$ ) suffice to produce a subdivision with similar properties, which would improve in practice the time and space complexity of the algorithm for many "real-life" polyhedra.

6. It seems to follow from Lemma 3.3 that an arbitrary polyhedron can be approximated by a self-conforming polyhedron: Slightly "shave off" or "flatten" any highdegree vertex, then use guaranteed mesh generation methods to convert facets to a new set of facets with fat triangles. It would be interesting to get an alternative $1+\epsilon$ approximation algorithm based on this observation.

7. Of course, the most interesting question is whether the shortest path map on a general nonconvex polyhedron can be (implicitly) constructed in time that is close to linear, or, at least, sub-quadratic. Alternatively, can this be achieved just for computing the shortest path between two specified points?

Acknowledgements I am very grateful to Micha Sharir, my adviser, for his invaluable help and support. I would like to thank Joe Mitchell for a thoughtful and thorough review of this paper, as well as Joseph O'Rourke and John Hershberger for their help in significant parts of this research. I am also grateful to Esther Moet for bringing the problem of shortest paths on realistic surfaces to my attention.

\section{References}

1. Agarwal, P.K., Katz, M.J., Sharir, M.: Computing depth orders for fat objects and related problems. Comput. Geom. Theory Appl. 5, 187-206 (1995)

2. Agarwal, P.K., Har-Peled, S., Sharir, M., Varadarajan, K.R.: Approximate shortest paths on a convex polytope in three dimensions. J. ACM 44, 567-584 (1997)

3. Aleksandrov, L., Maheshwari, A., Sack, J.-R.: An improved approximation algorithm for computing geometric shortest paths. In: Proc. of the 14th Internat. Sympos. Fundam. Comput. Theory (FCT'03). Lecture Notes in Computer Science, vol. 2751, pp. 246-257. Springer, Berlin (2003)

4. Alt, H., Fleischer, R., Kaufmann, M., Mehlhorn, K., Näher, S., Schirra, S., Uhrig, C.: Approximate motion planning and the complexity of the boundary of the union of simple geometric figures. Algorithmica 8, 391-406 (1992)

5. Chen, J., Han, Y.: Shortest paths on a polyhedron, Part I: Computing shortest paths. Int. J. Comput. Geom. Appl. 6, 127-144 (1996) 
6. de Berg, M.: Linear size binary space partitions for uncluttered scenes. Algorithmica 28, 353-366 (2000)

7. de Berg, M., Katz, M.J., van der Stappen, A.F., Vleugels, J.: Realistic input models for geometric algorithms. Algorithmica 34, 81-97 (2002)

8. Har-Peled, S.: Constructing approximate shortest path maps in three dimensions. SIAM J. Comput. 28(4), 1182-1197 (1999)

9. Hershberger, J., Suri, S.: An optimal algorithm for Euclidean shortest paths in the plane. SIAM J. Comput. 28(6), 2215-2256 (1999)

10. Kapoor, S.: Efficient computation of geodesic shortest paths. In: Proc. of the 32nd Annual ACM Sympos. Theory Comput., pp. 770-779 (1999)

11. Lanthier, M., Maheshwari, A., Sack, J.-R.: Approximating shortest paths on weighted polyhedral surfaces. Algorithmica 30(4), 527-562 (2001)

12. Mata, C., Mitchell, J.S.B.: A new algorithm for computing shortest paths in weighted planar subdivisions. In: Proc. of the 13th Annual ACM Sympos. Comput. Geom., pp. 264-273 (1997)

13. Mitchell, J.S.B., Mount, D.M., Papadimitriou, C.H.: The discrete geodesic problem. SIAM J. Comput. 16, 647-668 (1987)

14. Moet, E., van Kreveld, M., van der Stappen, A.F.: On realistic terrains. In: Proc. of the 22nd Annual ACM Sympos. Comput. Geom., pp. 177-186 (2006)

15. Mount, D.M.: On finding shortest paths on convex polyhedra. Technical report, Computer Science Dept., Univ. Maryland, College Park (October 1984)

16. Mount, D.M.: Storing the subdivision of a polyhedral surface. Discrete Comput. Geom. 2, 153-174 (1987)

17. O'Rourke, J., Suri, S., Booth, H.: Shortest paths on polyhedral surfaces. In: Proc. of the 2nd Sympos. Theoretical Aspects of Computer Science. Lecture Notes in Computer Science, vol. 182, pp. 243-254. Springer, London (1985)

18. Schreiber, Y.: Euclidean shortest paths on polyhedra in three dimensions. Ph.D. thesis (2007). http://www.cs.tau.ac.il/research/yevgeny.schreiber/SchreiberThesis.pdf

19. Schreiber, Y., Sharir, M.: An optimal-time algorithm for shortest paths on a convex polytope in three dimensions. Discrete Comput. Geom. (20th anniversary special issue) 39, 500-579 (2008), A full version available at http://www.math.tau.ac.il/ michas/ShortestPath.pdf. A preliminary version in Proc. of the 22nd Annual ACM Sympos. Comput. Geom., pp. 30-39 (2006)

20. Schwarzkopf, O., Vleugels, J.: Range searching in low-density environments. Inf. Process. Lett. 60, 121-127 (1996)

21. Sharir, M.: On shortest paths amidst convex polyhedra. SIAM J. Comput. 16, 561-572 (1987)

22. Sharir, M., Schorr, A.: On shortest paths in polyhedral spaces. SIAM J. Comput. 15, 193-215 (1986)

23. van der Stappen, A.F.: Motion planning amidst fat obstacles. Ph.D. thesis, Dept. Comput. Sci., Utrecht Univ., Utrecht, the Netherlands (1994)

24. van der Stappen, A.F., Overmars, M.H., de Berg, M., Vleugels, J.: Motion planning in environments with low obstacle density. Discrete Comput. Geom. 20(4), 561-587 (1998)

25. van Kreveld, M.: On fat partitioning, fat covering, and the union size of polygons. Comput. Geom. Theory Appl. 9, 197-210 (1998)

26. Varadarajan, K.R., Agarwal, P.K.: Approximating shortest paths on a nonconvex polyhedron. SIAM J. Comput. 30(4), 1321-1340 (2000) 